G.Y. SHER

\title{
PARTIALLY-ORDERED (BRANCHING) GENERALIZED QUANTIFIERS: A GENERAL DEFINITION*
}

\begin{abstract}
Following Henkin's discovery of partially-ordered (branching) quantification (POQ) with standard quantifiers in 1959, philosophers of language have attempted to extend his definition to POQ with generalized quantifiers. In this paper I propose a general definition of POQ with 1-place generalized quantifiers of the simplest kind: namely, predicative, or "cardinality" quantifiers, e.g., "most", "few", "finitely many", "exactly $\alpha$ ", where $\alpha$ is any cardinal, etc. The definition is obtained in a series of generalizations, extending the original, Henkin definition first to a general definition of monotone-increasing ( $\mathrm{M} \uparrow$ ) POQ and then to a general definition of generalized POQ, regardless of monotonicity. The extension is based on (i) Barwise's 1979 analysis of the basic case of M $\uparrow$ POQ and (ii) my 1990 analysis of the basic case of generalized POQ. POQ is a non-compositional 1st-order structure, hence the problem of extending the definition of the basic case to a general definition is not trivial. The paper concludes with a sample of applications to natural and mathematical languages.
\end{abstract}

\section{INTRODUCTION}

Quantifier-prefixes in standard logic are linearly ordered, both syntactically and semantically. By relaxing the demand that quantifier-prefixes be linearly ordered we obtain partially-ordered prefixes. While syntactically it is perfectly clear what a partially-ordered quantifier-prefix is like, the problem of devising a semantic definition of partially-ordered quantifications is still partly open. In his 1959 paper, "Some Remarks on Infinitely Long Formulas", Leon Henkin first introduced the concept of a partially-ordered (branching) quantification and solved its semantic problem for the standard quantifiers, $\forall$ and $\exists$. Henkin's solution is complete in the sense of applying to any partially-ordered quantifier-prefix, regardless of size, ordering, and the (standard) quantifiers involved. An

\footnotetext{
* I would like to thank Yair Guttmann, Charles Parsons, Peter Sher, Johan van Benthem, and two anonymous referees of the Journal of Philosophical Logic for contributing to the development of this paper. I am also thankful to the audiences at the 9th International Congress of Logic, Methodology and Philosophy of Science (Uppsala, 1991), the 1993 annual meeting of the Association of Symbolic Logic, and the CSLI Workshop on Logic, Language, and Computation (1993).
} 
example of a genuine Henkin sentence, i.e., one that is irreducible to a standard 1 st-order linear quantification, is

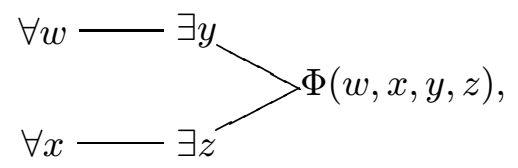

defined, semantically, by

$$
\exists f^{1} g^{1} \forall w x \Phi[w, x, f(w), g(x)] .
$$

(Here, and elsewhere, superscripts are displayed only in the first occurrence of a symbol.) Henkin's semantic definition is indirect: he identifies a branching 1st-order quantification ("1st-order" in the sense of having the same primitive vocabulary and variables as in standard 1st-order logic) with a linear 2nd-order quantification (2nd-order variables and quantifiers), whose semantic structure is well understood. I will refer to Henkin's partially-ordered standard quantifiers as PSQ.

In a 1973 paper, "Quantifiers vs. Quantification Theory", Jaakko Hintikka added a new dimension to the study of branching quantification. Hintikka noted that branching prefixes are found not only in artificial languages but also in natural languages, e.g., in the English sentence (p. 344)

Some relative of each villager and some relative of each townsman hate each other.

The two quantifier phrases "some relative of each villager" and "some relative of each townsman", Hintikka pointed out, are scope-wise independent, i.e., neither is within the scope of the other; therefore a nonlinear reading is required.

The problem of extending Henkin's definition from standard to generalized quantifiers has proven difficult and unwieldy. Even the problem of devising a semantics for partially-ordered quantification with the simplest type of generalized quantifiers, namely 1-place cardinality quantifiers, has resisted a complete solution. (1-place cardinality quantifiers were first introduced by Andrzej Mostowski in "On a Generalization of Quantifiers" (1957). Examples of Mostowski's quantifiers are "most", "few", "exactly three", "an even number of", "finitely many", etc. Henceforth I will call a partially-ordered quantification with generalized Mostowskian quantifiers a PGQ.)

In his 1979 paper, "On Branching Quantifiers in English", Jon Barwise made the first, ground breaking steps towards providing a semantics 
for PGQ. Barwise's work, however, goes only part way towards devising a complete and satisfactory semantics for PGQ: (A) His account is limited to very simple partial orderings. (B) His account is restricted to "homogeneous" quantifier-prefixes of a particular kind, namely those in which all the quantifiers are monotone increasing $(\mathrm{M} \uparrow)$ (e.g., "most", "at least two") and those in which all the (non-standard) quantifiers are monotone decreasing $(\mathrm{M} \downarrow)$ (e.g., "few", "at most two"). (C) His account is ununified in the sense of embodying two essentially different conceptions of the branching structure: one for $\mathrm{M} \uparrow$ branching quantifications and one for $\mathrm{M} \downarrow$ branching quantifications.

Barwise was aware that much work was yet to be done in the model theory of PGQ and several attempts to deal with (B) and (C) appear in the literature. In his 1987 paper, "Branching Generalized Quantifiers and Natural Language", Dag Westerståhl devised an ingenious method for putting Barwise's distinct definitions of $\mathrm{M} \uparrow$ and $\mathrm{M} \downarrow$ branching quantifications (plus another definition due to Johan van Benthem of branching quantifications with non-monotone (non-M) quantifiers of the form "exactly $n$ ") under one umbrella. But while Westerståhl's analysis unifies the existing Barwise (and van Benthem) definitions, as it stands, it is far from being a complete semantics for PGQ. In fact, neither (A), (B) nor (C) is fully remedied: the definition is still limited to several simple types of partial ordering; it excludes non-homogeneous quantifierprefixes and even homogeneous prefixes with such non-M quantifiers as "an even number of" and "three or seven"; and the truth conditions of branching quantifications with $\mathrm{M} \uparrow$ quantifiers are still intuitively different from those with $\mathrm{M} \downarrow$ quantifiers (or non-M quantifiers of the form "exactly $n$ ").

In "Ways of Branching Quantifiers" (1990a/1991a) I proposed a different approach to (B) and (C). Barwise's analysis posits an intimate connection between the monotonic properties of given quantifiers and the analysis of quantifier-prefixes in which they occur, but this purported connection is not supported by our experience with logical languages in general. In particular, in linear quantifier-languages we also have prefixes that differ in the monotonic features and composition of their quantifiers, yet the definition of linear prefixes (which fall under PGQ!) is not affected by these differences: " $\mathrm{Q}_{1} x_{1} \cdots \mathrm{Q}_{n} x_{n} \Phi x_{1} \cdots x_{n}$ " has the same semantic interpretation, no matter whether $\left\langle\mathrm{Q}_{1}, \ldots, \mathrm{Q}_{n}\right\rangle$ is monotonically homogeneous and whether the quantifiers involved are $\mathrm{M} \uparrow, \mathrm{M} \downarrow$, non-M of the form "exactly $n$ " or non-M of another form. This led me to question Barwise's disparate analyses of $\mathbf{M} \uparrow$ and $\mathbf{M} \downarrow$ branching quantifications. I proposed a general, unified definition of basic PGQ's applicable to 
all quantifiers, regardless of monotonicity (or continuity) and coinciding with Barwise's definition of branching M $\uparrow$ PGQ's when all the quantifiers involved are $\mathrm{M} \uparrow$. I also showed how, by generalizing certain features of this definition, we arrive at a family of branching structures, one of which exemplifies Barwise's conception of branching $\mathrm{M} \downarrow$ quantifiers (and one which essentially exemplifies van Benthem's conception of branching "exactly $n$ " quantifiers). ${ }^{1}$

No one, to the best of my knowledge, has offered a solution to (A). The main obstacle to creating a complete definition of PGQ is the failure of compositionality. Barwise expressed this point as follows:

... the discovery of branching quantification would force us to re-examine, and perhaps re-interpret, Frege's principle of compositionality according to which the meaning of a given expression is determined by the meanings of its constituent phrases. ... the meaning of a branching quantifier expression of logic ... cannot be defined inductively in terms of simpler formulas, by explaining away one quantifier at a time. Rather the whole block [of quantifiers in a given prefix] must be treated at once. This has obvious consequences for any attempt to capture the relation between the syntax and semantics of ... sentences in which branching quantification occurs. (1979, p. 47).

The task is particularly difficult in the case of branching generalized quantifications, since in addition to the fact that the structure of PGQ's is not inductive (and the usual method of a recursive definition of truth is not applicable) there are no theorems establishing quantifier equivalences for generalized quantifiers like the ones for standard quantifiers that support Henkin's functional definition of PSQ.

In this paper I would like to attempt a complete semantic definition of PGQ, based on the analysis of the general basic case of PGQ in Sher (1990a/1991a). Although the definition will not be compositional in the usual sense, it will consist of an algorithm for translating a 1storder PGQ into a 2nd-order linear quantification in a finite number of steps. In Sher (1990a/1991a) I delineated a "family" of partially-ordered quantifier-structures, but here I will limit myself to a single structure, one which naturally extends Henkin's PSQ and Barwise's basic case of $\mathrm{M} \uparrow$ branching quantification.

\section{Methodological Guidelines}

In constructing a general definition of a given concept there is, obviously, no absolute question of right and wrong. There are many ways to systematize an idea, and a general definition is, in a sense, a proposal of how to apply the idea uniformly in a certain context. This is all the more so when the defined concept is new and there is no accepted tradition of applications. (Indeed, in the case of PGQ there is no data at all on the meaning of quantifications with relatively complex prefixes.) My 
definition of PGQ is, however, an attempt to generalize certain existent approaches to quantification, and these approaches dictate the following guidelines:

(A) The general definition of PGQ shall coincide with Henkin's general definition of PSQ when the quantifiers involved are standard.

(B) The general definition of PGQ shall coincide with the general definition of generalized linear quantification when the quantifier prefix is linear.

(C) The general definition of PGQ shall coincide with Barwise's definition of the basic case of $\mathrm{PM} \uparrow \mathrm{Q}$ (partially-ordered $\mathrm{M} \uparrow$ quantifiers) when the quantification is a basic $\mathrm{PM} \uparrow \mathrm{Q}$.

(D) The general definition PGQ shall coincide with the definition of the basic case of PGQ in Sher (1990a/1991a) when the quantification is a basic PGQ.

(E) Whenever (A)-(D) leave more than one option open, the definition shall be as simple and as common-sensical as possible.

This is my starting point. I take (A)-(D) as recommendations, to be upheld or given up depending on whether they lead to an intuitively satisfactory definition. Other definitions are, of course, not ruled out. Such definitions may be co-extensional with the one proposed here or they may capture other concepts of partially-ordered quantification.

Because of the complex nature of the enterprise and the novelty of the attempt, my goal in this paper is not to establish mathematical or linguistic results, but rather to lay down as clearly as possible the intuitions that led me to the final definition. To this end I will construct the general definition of PGQ in a succession of three generalizations, starting with the familiar constructions of Skolem forms and Henkin prefixes and showing how, by generalizing certain features of these constructions, we arrive first at the general structure of $\mathrm{PM} \uparrow \mathrm{Q}$, and from there at the general structure of PGQ. While Skolem forms are well known, my algorithmic account will bring out features that are usually left implicit and it will serve as a prototype for the more complicated algorithms that follow. More generally, each of the first three definitions will be formulated in such a way as to serve as a basis for the next generalization. For Henkin's definition I will use the method outlined in Walkoe (1970); I will also employ this method in my account of Skolem transformations. My general definition of $\mathrm{PM} \uparrow \mathrm{Q}$ employs a relational method, similar to that used by Marcin Mostowski (1987) for PSQ, though developed independently of his. While Walkoe's theorems allowed Mostowski to use a relatively simple relational representation of PSQs, no parallel theorems for PGQ were available to me (indeed, there was no general semantic notion of 
PGQ to have theorems for!) and my method is, therefore, more complex. The clue to getting from $\mathrm{PM} \uparrow \mathrm{Q}$ to $\mathrm{PGQ}$ is a maximality condition that was introduced, for the simple case, in Sher (1990a/1991a). I will explain this and other relevant issues from the literature as I go along.

The progression leading to the general definition of PGQ can be outlined by means of a simple example (see Sher, 1994):

(A) Skolem form:

$$
\forall w \exists y \forall x \exists z \Phi w x y z=\text { Df } \exists f^{1} g^{2} \forall w x \Phi[w, x, f(w), g(w, x)] .
$$

(B) PSQ (Henkin):

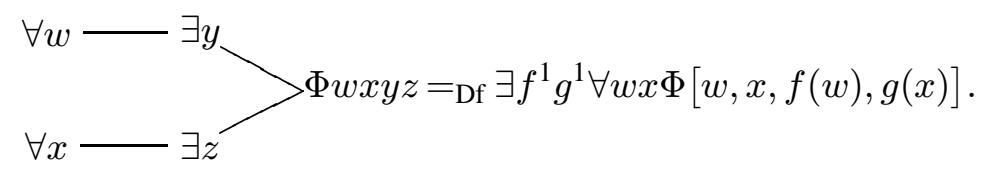

(C) $P M \uparrow Q$ (extension of Barwise): Let $\mathrm{Q}_{1}-\mathrm{Q}_{4}$ be any $\mathrm{M} \uparrow$ generalized quantifiers,

$$
\begin{aligned}
\mathrm{Q}_{2} x & \mathrm{Q}_{3} y \\
= & \mathrm{Q}_{4} z \\
{ }_{\mathrm{Df}} & \exists \mathrm{W}^{1} \mathrm{X}^{1} \mathrm{Y}^{2} \mathrm{Z}^{2}\left[\mathrm{Q}_{1} w \mathrm{~W} w \& \mathrm{Q}_{2} x \mathrm{X} x\right. \\
& \& \forall w\left(\mathrm{~W} w \rightarrow \mathrm{Q}_{3} y \mathrm{Y} w y\right) \& \forall x\left(\mathrm{X} x \rightarrow \mathrm{Q}_{4} z \mathrm{Z} x z\right) \\
& \& \forall w x y z(\mathrm{Y} w y \& \mathrm{Z} x z \rightarrow \Phi w x y z)]
\end{aligned}
$$

(D) $P G Q$ (extension of Sher): let $\mathrm{Q}_{1}-\mathrm{Q}_{4}$ be any generalized quantifiers,

$$
\begin{aligned}
\mathrm{Q}_{2} x & -\mathrm{Q}_{3} y-\mathrm{Q}_{4} z \\
& ={ }_{\mathrm{Df}} \exists \mathrm{W}^{1} \mathrm{X}^{1} \mathrm{Y}^{2} \mathrm{Z}^{2}\left[\mathrm{Q}_{1} w \mathrm{~W} w \& \mathrm{Q}_{2} x \mathrm{X} x\right.
\end{aligned}
$$

$\& \mathrm{~W}$ is a max. set s.t. $\forall w\left(\mathrm{~W} w \rightarrow \mathrm{Q}_{3} y \mathrm{Y} w y\right)$

$\& \mathrm{X}$ is a max. set s.t. $\forall x\left(\mathrm{X} x \rightarrow \mathrm{Q}_{4} z \mathrm{Z} x z\right)$

$\&\langle\mathrm{Y}, \mathrm{Z}\rangle$ is a max. pair s.t.

$\forall w x y z(\mathrm{Y} w y \& \mathrm{Z} x z \rightarrow \Phi w x y z)]$.

Linguistically: Henkin's definition allows us to account for statements like Hintikka's (3). Barwise's definition of the basic case of PM $\uparrow \mathrm{Q}$ provides a semantic analysis of Barwise (1979, p. 60; my emphasis)

Quite a few boys in my class and most girls in your class have all dated each other. 
My definition of the basic case of PGQ in "Ways of Branching Quantifiers" applies to

An even number of dots and an odd number of stars are all connected by lines,

Mots of my friends have all applied to the same few graduate programs

(1990a, p. 414 / 1991a, p. 123; new emphasis). The general definition of PGQ below will allow us to analyze more complex English branching quantifications, e.g.,

Two boys have two toys and two friends who don't like their toys,

Two veterans have two friends and two foes who fought in the same two wars.

(The repeated use of "two" is not essential. Any combination of generalized quantifiers will work, although in some cases the 2-place version of quantifiers will be required.)

\section{SKOLEM TRANSFORMATIONS}

The Skolem Normal Form Theorem says that every standard 1st-order well-formed formula (henceforth, wff), $\Psi$, is logically equivalent to a 2nd-order wff of the form

$$
\exists f_{1} \cdots f_{m} \forall x_{1} \cdots x_{n} \Phi
$$

where $f_{1}, \ldots, f_{m}$ are functional variables (a 0 -place function is an individual), $x_{1}, \ldots, x_{n}$ are individual variables, $n, m \geqslant 0$, and $\Phi$ is a quantifier-free wff.

Here I am interested in a weaker version of the Skolem Normal Form theorem which I will call the Skolem Form Theorem: Every standard 1storder quantificational wff $\Psi$ (i.e., non-atomic wff of standard 1st-order logic whose main logical operator is a quantifier) is logically equivalent to a 2 nd-order wff of the form (9), where $f_{1}, \ldots, f_{m}$ and $x_{1}, \ldots, x_{n}$ are as above, $m>0$ or $n>0$, and $\Phi$ is a non-quantificational wff (i.e., the main logical operator of $\Phi$, if any, is not a quantifier). (9) - as defined in the Skolem Form Theorem - is a Skolem form, and the functions satisfying a Skolem form are Skolem functions. 
The idea is that instead of saying "For every $x$ there is a $y$ such that $\Phi x y$ ", we can say: "There is a function $f$ which assigns to each $x$ a value $y$ such that $\Phi x y$ ". Applying this principle systematically, we can transform any 1st-order quantificational wff into an equivalent Skolem form. For example, we transform

$$
\forall w \exists y \forall x \exists z \Phi w x y z
$$

into

$$
\exists f^{1} g^{2} \forall w x \Phi[w, x, f(w), g(w, x)] .
$$

I will now describe a general procedure for transforming any standard 1 st-order quantificational wff of the form

$$
\mathrm{Q}_{1} x_{1}, \ldots, \mathrm{Q}_{n} x_{n} \Phi\left(x_{1}, \ldots, x_{n}\right),
$$

where $n \geqslant 1$ and $x_{1}, \ldots, x_{n}$ occur free in $\Phi,{ }^{2}$ into a logically equivalent 2nd-order Skolem form.

(Syn-0) - Syntax

Let $\mathrm{L}$ be a standard 1st-order language, with vocabulary as, for example, in Enderton (1972). We define a quantifier-prefix in L to be a system,

$$
Q=\left(\left\{q_{1}, \ldots, q_{n}\right\},>\right),
$$

where

(i) $n$ is a positive integer;

(ii) for $1 \leqslant i \leqslant n$, there is an individual variable $x_{i}$ such that $q_{i}=\exists x_{i}$ or $q_{i}=\forall x_{i}$;

(iii) for $1 \leqslant i \neq j \leqslant n, x_{i} \neq x_{j}$;

(iv) $>$ is a strong linear ordering of $\left\{q_{1}, \ldots, q_{n}\right\}$.

Note that the definition of $Q$ is not compositional. ${ }^{3}$

\section{Definitions relative to $Q$ :}

1. $\exists$ and $\forall$ are quantifiers: the existential quantifier and the universal quantifier, respectively. $\exists x_{i}$ and $\forall x_{i}$ are quantifier atoms: an existential quantifier atom and a universal quantifier atom, respectively. (Outside the definitions of the syntax and the semantics I will use "quantifier" both for "quantifier" and for "quantifier atom". It will be clear from the context what I am referring to.)

2. If $q_{i}>q_{j}$, then $q_{i}$ governs $q_{j}$ and $q_{j}$ is dependent on $q_{i}$. If $q_{i}$ is a universal quantifier governing $q_{j}$, we say that $q_{i}$ is a universal governor of $q_{j}$. 
3. The rank of an existential quantifier, $q_{i}, r\left(q_{i}\right)$, or simply $r_{i}$, is the number of universal governors of $q_{i}$.

Method of syntactic representation:

If $q_{i}>q_{j}$, then $q_{i}$ is to the left of $q_{j}{ }^{4}$

Definition of a wff: Same as in Enderton (1972), except that the entry for quantified wffs is replaced by "If $Q$ is a quantifier-prefix and $\Phi$ is a wff, then $(Q \Phi)$ is a wff."

(Sem-0) - Semantics

Let $\mathrm{L}^{\prime}$ be a standard 2nd-order language with syntax and semantics as, for instance, in Enderton (1972). Assume $L \subset L^{\prime}$.

Transformation of standard 1st-order quantifications (L) into Skolem forms $\left(\mathrm{L}^{\prime}\right)$.

Let $Q=\left(\left\{q_{1}, \ldots, q_{n}\right\},>\right)$ be a quantifier-prefix of $\mathrm{L}$, and let $\Phi\left(x_{1}\right.$, $\left.\ldots, x_{n}\right)$ be a wff of L. With each existential quantifier atom $q_{i}$ in $Q$ we associate

(a) a distinct functional variable of $\mathrm{L}^{\prime}$,

$$
f_{i}^{r_{i}}
$$

the arity of which is $r_{i}$ (the rank of $q_{i}$ ); and

(b) a functional term of $\mathrm{L}^{\prime}$,

$$
\mathrm{F}_{i}=f_{i}^{r_{i}}\left(x_{i_{1}}, \ldots, x_{i_{r_{i}}}\right),
$$

where $q_{i_{1}}, \ldots, q_{i_{r_{i}}}$ are all the universal governors of $q_{i}$. We can say that $F_{i}$ traces the "essential", i.e., universal, governors of the existential quantifier atom $q_{i}$ through its arguments, $x_{i_{1}}, \ldots, x_{i_{i}}$, or that $\mathrm{F}_{i}$ represents the (essential) dependencies of $q_{i}$ (i.e., $F_{i}$ displays the dependency relation in which $q_{i}$ stands to its "essential" governors). We define the Skolem transformation of $Q \Phi\left(x_{1}, \ldots, x_{n}\right)$ as follows:

$$
\begin{aligned}
& Q \Phi\left(x_{1}, \ldots, x_{n}\right) \text { IS TRANSFORMED INTO } \\
& \exists f_{i_{1}}^{r_{i_{1}}} \cdots f_{i_{m}}^{r_{i_{m}}} \forall x_{j_{1}} \cdots x_{j_{k}} \Phi\left(s_{1}, \ldots, s_{n}\right),
\end{aligned}
$$

where

(i) $q_{i_{1}}, \ldots, q_{i_{m}}$ are all the existential quantifier atoms in $Q$,

(ii) $\forall x_{j_{1}}, \ldots, \forall x_{j_{k}}$ are all the universal quantifier atoms in $Q$,

(iii) for $1 \leqslant h \leqslant n, s_{h}$ replaces every free occurrence of $x_{h}$ in $\Phi$, where

$$
s_{h}= \begin{cases}x_{h} & \text { if } q_{h}=\forall x_{h} \\ F_{h} & \text { if } q_{h}=\exists x_{h} .\end{cases}
$$


Remark. The transformation procedure is unique up to logical equivalence. Among the inessential variations it allows are: (i) changes in the order of consecutive existential quantifiers both in $Q$ and in its 2nd-order correlate; (ii) changes in the order of consecutive universal quantifiers in both prefixes; (iii) changes in the order of variables in the functional terms assigned to the existential quantifiers in $Q$ (but it is essential that the order of variables in repeated occurrences of a given functional term remain the same).

The Skolem Form Theorem says that (ST) transforms $Q \Phi\left(x_{1}, \ldots, x_{n}\right)$ into a logically equivalent formula. Based on it we construct a 2nd-order definition of satisfaction for $\mathrm{L}$.

Terminology: If $Q$ is a quantifier-prefix of $\mathrm{L}$ and $\Phi$ is a wff of $\mathrm{L}$, then $\mathrm{T}(Q \Phi)$ is the Skolem Transformation of $Q \Phi$.

Definition of $\mathrm{T}^{*}(\Psi)$, where $\Psi$ is a wff of $\mathrm{L}$ :

(a) If $\Psi$ is an atomic wff, then $\mathrm{T}^{*}(\Psi)=\Psi$.

(b) If $\Psi=(\sim \Phi)$, then $\mathrm{T}^{*}(\Psi)=\sim\left(\mathrm{T}^{*}(\Phi)\right)$.

(c) If $\Psi=(\Phi \rightarrow \Upsilon)$, then $\mathrm{T}^{*}(\Psi)=\left(\mathrm{T}^{*}(\Phi) \rightarrow \mathrm{T}^{*}(\Upsilon)\right)$.

(d) If $\Psi=(Q \Phi)$, then $\mathrm{T}^{*}(\Psi)=\mathrm{T}(Q \Phi)$.

Let $\Psi$ be a wff of $\mathrm{L}, \mathfrak{U}$ a model for $\mathrm{L}$ (the notion of model is the standard one) and $g$ an assignment function for the variables of $L$ in $\mathfrak{U}$.

Definition of satisfaction for $\mathrm{L}: \mathfrak{U}$ satisfies $\Psi$ with $g$ iff $\mathfrak{U}$ satisfies $\mathrm{T}^{*}(\Psi)$ with $g^{*}$, where $g^{*}$ is any extension of $g$ to the 2 nd-order variables of $\mathrm{L}^{\prime}$.

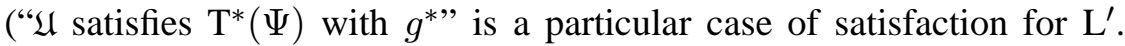
See Enderton, 1972.)

\section{PARTIALLY-ORDERED STANDARD QUANTIFIERS (PSQ) - HENKIN}

To arrive at Henkin's quantifiers we introduce one change into the syntactic system presented in Section 2: we relax the requirement that $>$ be a strong linear ordering, demanding that $>$ be a strong partial ordering instead. We also amend the method of syntactic representation by instituting the rule that if $q_{i}$ is an immediate governor of $q_{j}$ (i.e., there is no $q_{k}$ such that $q_{i}>q_{k}>q_{j}$ ), then there is a straight line connecting $q_{i}$ and $q_{j}$. If $q_{i}$ is a smallest element in the ordering, then a straight line connects $q_{i}$ to the quantified formula. In this way we obtain a new logical form: 
that of partially-ordered standard quantification, PSQ. An example of a PSQ is

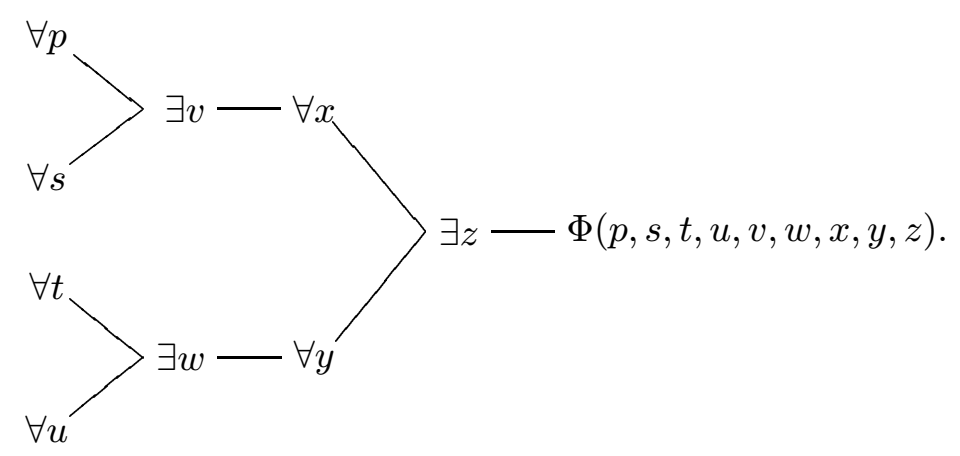

What do PSQ's mean? The transformation procedure of standard 1storder formulas into Skolem forms establishes a systematic connection between existential quantifiers and Skolem functions, a connection that is based on dependencies of existential upon universal quantifiers. Since the partially-ordered quantifier-prefix also exhibits such dependencies, the same procedure can be used to create Skolem forms of PSQ's. Here, however, the transformation method has a different role: Since we do not possess an independent understanding of the new quantificational form, we use the transformation procedure as a semantic definition of the new form. I.e., we use the transformation procedure as a 2nd-order definition of the 1st-order partially-ordered quantificational form. Since the 2nd-order formula correlated with a given PSQ is itself well understood (having a linear structure), it can properly be used as a definiens. Thus, by applying the Skolem procedure to the partially-ordered (13) we obtain a linear interpretation, namely

$$
\begin{aligned}
& \exists f^{2} g^{2} h^{6} \forall p s t u x y \\
& \quad \Phi[p, s, t, u, f(p, s), g(t, u), x, y, h(p, s, t, u, x, y)] .
\end{aligned}
$$

The main difference between Skolem forms for linear quantifications and those for partially-ordered quantifications is the following:

When $\exists f_{1} \cdots f_{m} \forall x_{1} \cdots x_{n} \Phi$ is a Skolem form of a linear quantification, its existential sub-prefix can be ordered in such a way that if $f_{i}$ is to the left of $f_{j}$, then the set of arguments of $f_{i}$ in $\Phi$ is essentially included in the set of arguments of $f_{j}$ in $\Phi$. But, in general, this cannot be done when $\exists f_{1} \cdots f_{m} \forall x_{1} \cdots x_{n} \Phi$ is a Skolem form of a non-linear quantification. (Based on Henkin 1959, p. 181.)

So, in the Skolem correlate of the linear (10) the arguments of $f$ are included among the arguments of $g$, but in the Skolem correlate of the partially-ordered (13) neither the arguments of $f$ are included among 
the arguments of $g$, nor the other way around. The simplest PSQ not equivalent to any linear 1st-order quantification is of the form

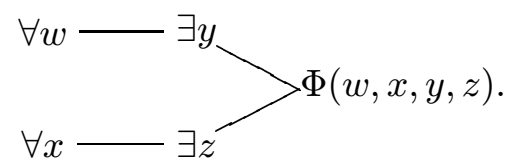

Its Skolem correlate is

$$
\exists f^{1} g^{1} \forall w x \Phi[w, x, f(w), g(x)] .
$$

(Syn-1)

The syntax of PSQ, (Syn-1), is obtained from (Syn-0) by replacing (iv) in the definition of quantifier-prefix by:

(iv) $^{*}>$ is a strong partial ordering of $\left\{q_{1}, \ldots, q_{n}\right\}$.

(Sem-1)

The semantics of PSQ, (Sem-1), is essentially the same as (Sem-0). (We restrict the assumption that $\mathrm{L} \subset \mathrm{L}^{\prime}$ to the linear part of $\mathrm{L}$. The notion of model is the same as before.)

Linguistic Applications. Hintikka (1973) pointed out that some natural language quantifications have the form of a PSQ. A well known example due to Hintikka is

Some relative of each villager and some relative of each townsman hate each other.

This sentence, Hintikka pointed out, contains two pairs of quantifiers, the quantifiers in each pair being independent of the quantifiers in the other. A linear reading would create a dependency of one pair of quantifiers on the other, but a partially-ordered reading correctly simulates the dependencies and independencies involved. On Hintikka's reading, (3) has the syntactic form of (1) and the meaning of (2).

In "Ways of Branching quantifiers" I pointed out that sentences of the form (1) imply the existence of a "massive nucleus" of objects satisfying the relation $\Phi$. In the case of Hintikka's (3), the reading requires a massive nucleus of villager relatives and townsman relatives hating each other: at least one relative of each villager and at least one relative of each townsman belong in the nucleus; each villager relative in the nucleus hates all the townsman relatives in it, and each townsman relative in the nucleus hates all the villager relatives in it. I called the hatred relation between villager relatives and townsman relatives in Hintikka's (3) an 
"each-all relation", or a "Cartesian product relation", and I noted that each-all relations (or variants thereof) are typical of Henkin-like nonlinear quantifications.

Graphically, we can represent the statement made by (3) as follows:

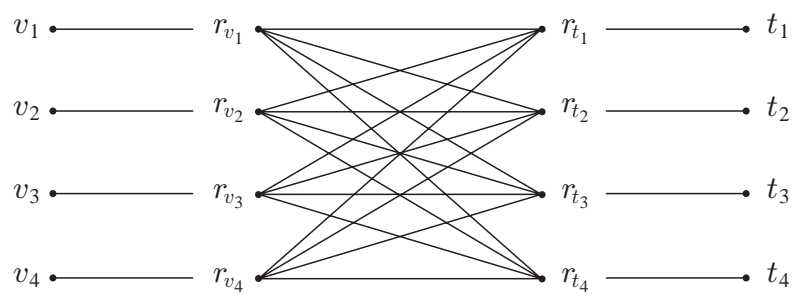

Graph I

The meaning of (3) is perhaps more clearly exhibited in the following paraphrase:

Every villager has a relative and every townsman has a relative who all hate each other.

While (3) ((15)) has the form "[QP] and [QP] 〈predicate〉", where [QP] stands for a quantifier-prefix, genuine branching quantifications in English, Hintikka says, are not restricted to this form. Thus,

Some reviewer of every newspaper admires some book of every publisher ${ }^{5}$

includes two scopally independent quantifier-prefixes, and hence is a genuine branching quantification, though its form is " $[\mathrm{QP}]\langle$ predicate $\rangle[\mathrm{QP}]$ ".

\section{PARTIALLY-ORDERED M $\uparrow$ QUANTIFIERS (PM $\uparrow \mathrm{Q})-$ EXTENSION OF BARWISE}

Henkin's definition applies to partially-ordered quantifier prefixes with the standard quantifiers, $\forall$ and $\exists$. But with the generalization of the standard quantifiers by Andrzej Mostowski and Per Lindström (A. Mostowski, 1957; Lindström, 1966) an interest in extending Henkin's definition to the new quantifiers naturally arose. The generalized quantifiers I will consider in this paper are unary Mostowskian quantifiers. Semantically, these quantifiers can be seen as functions from sets of objects in a given universe to a truth value. Intuitively, (logical) quantifiers do 
not distinguish the identity of individuals in a given universe. Model theoretically, quantifiers are invariant under isomorphic set structures (structures of the form $\langle\mathrm{A}, \mathrm{B}\rangle$, where $\mathrm{B} \subseteq \mathrm{A}$ ). Mostowski showed that functions satisfying this invariance condition take only the cardinalities of B and its complement in A into account. Examples of generalized (Mostowskian) quantifiers are "most" (understood here, for the sake of simplicity, as "more than half"), "few" (understood here as "less than half"), "exactly ten", "infinitely many", and "an even number of". Using Mostowski's style of definition, we can describe the semantics of these quantifiers as follows: Each quantifier $\mathrm{Q}$ is associated with a function, $\mathfrak{g}$, over sets (universes of models) such that if $\mathrm{A}$ and $\mathrm{A}^{\prime}$ are sets of the same cardinality $-|A|=\left|\mathrm{A}^{\prime}\right|-$ then $\mathfrak{g}(\mathrm{A})=\mathfrak{g}\left(\mathrm{A}^{\prime}\right)$. Given a universe $\mathrm{A}, \mathfrak{g}(\mathrm{A})$ is itself a function, $q$, from pairs of cardinal numbers $\alpha, \beta$ such that $\alpha+\beta=|\mathrm{A}|$ into $\{\mathrm{T}, \mathrm{F}\}$. Intuitively, given a 1-place predicate $\mathrm{P}$, the pairs $(\alpha, \beta)$ such that $q(\alpha, \beta)=\mathrm{T}$ represent the numerical combinations of objects satisfying $\mathrm{P}$ and objects not satisfying P "permitted" by Q. If $\mathfrak{U}$ is a model with a universe A, we can describe the truth conditions of a quantification " $\mathrm{Q} x \mathrm{~B} x$ " in $\mathfrak{U}$ by:

$$
\text { "Q } x \Phi x " \text { is true in } \mathfrak{U} \text { iff } q\left(\left|\Phi^{\mathfrak{U}}\right|,\left|\mathrm{A}-\Phi^{\mathfrak{U}}\right|\right)=\mathrm{T},
$$

where $\Phi^{\mathfrak{U}}$ is the extension of $\Phi$ in $\mathfrak{U}$. The functions associated with the quantifiers mentioned above can be defined as follows: $\operatorname{most}(\alpha, \beta)=\mathrm{T}$ iff $\alpha>\beta$; few $(\alpha, \beta)=\mathrm{T}$ iff $\alpha<\beta$; exactly ten $(\alpha, \beta)=\mathrm{T}$ iff $\alpha=10$; infinitely many $(\alpha, \beta)=\mathrm{T}$ iff $\alpha \geqslant \aleph_{0}$; an even number of $(\alpha, \beta)=\mathrm{T}$ iff $\alpha$ is an even number. The standard quantifiers, $\forall$ and $\exists$, can also be defined in this manner: $\operatorname{all}(\alpha, \beta)=\mathrm{T}$ iff $\beta=0$; some $(\alpha, \beta)=\mathrm{T}$ iff $\alpha>0$.

The first to extend Henkin's definition to partially-ordered generalized quantifiers was Jon Barwise in "On Branching Quantifiers in English" (1979). Barwise exploited a certain similarity between the standard quantifiers and some of the (non-standard) generalized quantifiers to provide a partial definition of PGQ. The quantifiers in question all share the property of being monotone increasing, $\mathrm{M} \uparrow$. (A quantifier $\mathrm{Q}$ is $\mathrm{M} \uparrow$ iff for any wffs $\Phi$ and $\Psi$, "Q $x \Phi x \& \forall x(\Phi x \rightarrow \Psi x)$ " implies "Q $\mathrm{Q} \Psi \Psi x$ ".) Among the generalized quantifiers (other than $\forall$ and $\exists$ ) possessing this property are "most", "at least ten", "infinitely many", and "quite a few". Barwise $(1979$, p. 63) proposed the following definition of the two basic cases of $\mathrm{PM} \uparrow \mathrm{Q}$ : Let $\mathrm{Q}_{1}$ and $\mathrm{Q}_{2}$ be $\mathrm{M} \uparrow$ quantifiers;

$$
\mathrm{Q}_{1} x \Phi x={ }_{\mathrm{Df}} \exists X^{1}\left[\mathrm{Q}_{1} x \mathrm{X} x \& \forall x(\mathrm{X} x \rightarrow \Phi x)\right],
$$




$$
\begin{array}{r}
\mathrm{Q}_{1} x>\Phi x y={ }_{\mathrm{Df}} \exists \mathrm{X}^{1} \mathrm{Y}^{1}\left[\mathrm{Q}_{1} x \mathrm{X} x \& \mathrm{Q}_{2} y \mathrm{Y} y\right. \\
\& \forall x y(\mathrm{X} x \& \mathrm{Y} y \rightarrow \Phi x y)] .
\end{array}
$$

We can paraphrase (18) informally as: " $\mathrm{Q}_{1} x$ and $\mathrm{Q}_{2} y$ all stand in the relation $\Phi$ ". Note that just as in the case of Henkin's quantifiers, (18) includes an "each-all" condition. What (18) says is: There is a group with $\mathrm{Q}_{1}$ elements, and there is a group with $\mathrm{Q}_{2}$ elements, such that each member of the first group stands in the relation $\Phi$ to all the elements of the second group. Two English sentences exemplifying (18) are:

Most philosophers and most linguists agree with each other about branching quantification (Barwise 1979, p. 60);

Quite a few boys in my class and most girls in your class have all dated each other.

The precise form of these sentences involves binary quantifiers. (Binary Mostowskian quantifiers are based on the same principle as unary Mostowskian quantifiers: they are defined by binary functions from pairs of sets in a given universe to truth values, taking only cardinalities into account.) ${ }^{6}$ I will not discuss binary branching quantification here (see Sher 1990a/1991a), but even without a detailed analysis the examples should give an intuitive idea of what PM $\uparrow$ 's mean. Thus, (4) says that there is a group with quite a few boys from my class, and there is a group with most girls from your class, such that each boy in the first group has dated (and has been dated by) all the girls in the second group. Note the occurrence of the inner quantifier expression "all" in (4): "all" stands for "each-all". In (19), on the other hand, the each-all condition is only implicit, having no syntactic representation. In this respect (19) is more similar to linear quantifications, where the relations between the various components are usually implicit. (The implicit relation between the two quantifiers in " $\mathrm{Q}_{1} x \mathrm{Q}_{2} y \Phi x y$ " is "for each one of which": "There are $\mathrm{Q}_{1}$ things $x$ for each one of which there are $\mathrm{Q}_{2}$ things $y$ such that $\Phi x y$ ".)

Barwise did not extend his definition beyond the basic cases, so it is left for us to complete his definition. (Recall that a simple recursion based on the structure of PM $\uparrow Q$ 's is not available here.) I will begin by analyzing the difference between Henkin's and Barwise's quantifiers. Henkin limited himself to two quantifiers, $\forall$ and $\exists$. Prefixes with these quantifiers exhibit four basic types of dependency: $\langle\exists x, \exists y\rangle$, $\langle\forall x, \forall y\rangle,\langle\exists x, \forall y\rangle$ and $\langle\forall x, \exists y\rangle$. The first three dependencies are inessential. It so happens that the only essential dependency with standard 
quantifiers - i.e., $\langle\forall x, \exists y\rangle$ or, more generally, $\left\langle\forall x_{1}, \ldots, \forall x_{n}, \exists y\right\rangle-$ is functional: " $\forall x_{1} \cdots x_{n} \exists y \Phi\left(x_{1}, \ldots, x_{n}, y\right)$ " is logically equivalent to " $\exists f^{n} \forall x_{1} \cdots x_{n} \Phi\left(x_{1}, \ldots, x_{n}, f\left(x_{1}, \ldots, x_{n}\right)\right)$ ". This particular feature of the standard dependencies explains Henkin's use of Skolem functions in his definition. But when the context extends to $\mathrm{M} \uparrow$ quantifiers in general, the situation is different. Here we have a large variety of essential dependencies, functional and non-functional. (An essential non-functional dependency appears in "(Most $x)($ Most $y) \Phi x y$ ".) It follows that Skolem functions cannot be used in extending Henkin's definition to generalized quantifiers. We can, however, treat functions as a special kind of relations, and the idea comes to mind of replacing the Skolem functions in Henkin's definition by relational terms. This will allow us to renounce the distinction between functional and non-functional dependencies and treat all dependencies - essential and inessential - on a par.

Based on these observations I will now show how Henkin's definition gives rise to Barwise's and how a general definition of $\mathrm{PM} \uparrow \mathrm{Q}$ based on Barwise's principles can be arrived at. An adequate definition of $\mathrm{PM} \uparrow \mathrm{Q}$ should (a) coincide with Henkin's definition when all quantifiers are standard, (b) coincide with the usual definition of linear quantification when the partial ordering is linear, and (c) coincide with Barwise's definitions (17) and (18) over the basic cases. I will proceed as follows: First I will show, by means of an example, how to transform Henkin's definition to a definition based on relational terms instead of Skolem functions. Next, I will show, again using examples, how to transform the usual semantics of $\mathrm{M} \uparrow$ linear quantification to a 2nd-order relational definition. (The simplest case is (17).) Finally, I will motivate Barwise's definition of the simplest case of a genuine (non-linear) PM $\uparrow \mathrm{Q}$, (18). Following these steps, I will present a general definition of PM $\uparrow \mathrm{Q}$ based on Barwise's principles.

Transformation of Henkin's definition of a PSQ to a relational definition. ${ }^{7}$ Consider Henkin's definiens of (1), namely

$$
\exists f^{1} g^{1} \forall w x \Phi[w, x, f(w), g(x)] .
$$

Our present task is to transform (2) into an equivalent statement in which relational terms replace functional terms and all the 1st-order quantifiers are treated on a par (i.e., there is no distinction between essential and inessential dependencies). We proceed in two steps:

(i) We replace $f^{1}$ and $g^{1}$ by binary relational variables and obtain a formula equivalent to (2): 


$$
\begin{aligned}
& \exists \mathrm{Y}^{2} \mathrm{Z}^{2}[\forall w \exists y \mathrm{Y} w y \& \forall x \exists z \mathrm{Z} x z \\
& \quad \& \forall w x y z(\mathrm{Y} w y \text { \& } \mathrm{Z} x z \rightarrow \Phi w x y z)] .
\end{aligned}
$$

(ii) We assign to $\forall w$ and $\forall x$ relational variables and formulas (as we did to $\exists y$ and $\exists z$ ), and we transform (20) to the equivalent

$$
\begin{aligned}
& \exists \mathrm{W}^{1} \mathrm{X}^{1} \mathrm{Y}^{2} \mathrm{Z}^{2}[\forall w \mathrm{~W} w \& \forall x \mathrm{X} x \& \forall w(\mathrm{~W} w \rightarrow \exists y \mathrm{Y} w y) \\
& \quad \& \forall x(\mathrm{X} x \rightarrow \exists z \mathrm{Z} x z) \\
& \quad \& \forall w x y z(\mathrm{Y} w y \& \mathrm{Z} x z \rightarrow \Phi w x y z)] .
\end{aligned}
$$

Thus (21) is equivalent to (2). (In the first step the axiom of choice is used.)

How do we get directly from (1) to (21)? - First, we assign ranks not just to the existential quantifiers but to all the quantifiers in (1), modifying the principle of ranking to adjust for the fact that an $n$-ary function is an $n+1$-ary relation. I.e., given a quantifier $q, r(q)=$ the number of governors of $q+1$. Thus: $r(\forall w)=(\forall x)=1$ and $r(\exists y)=r(\exists z)=2$. Second, we assign to each quantifier $q$ a relational variable of arity $r(q)$ and a relational formula which traces its dependencies (if any). Namely: $\forall w-\mathrm{W}^{1}, \mathrm{~W} w ; \forall x-\mathrm{X}^{1}, \mathrm{X} x ; \exists y-\mathrm{Y}^{2}, \mathrm{Y} w y$; and $\exists z-\mathrm{Z}^{2}, \mathrm{Z} x z$. Next, we specify the conditions set by the quantifiers in (1) on each relational variable: $\mathrm{W}$ and $\mathrm{X}$ are universal, $\mathrm{Y}$ and $\mathrm{Z}$ include functional relations with domains $\mathrm{W}$ and $\mathrm{X}$ respectively. Finally, we state that $\Phi$ includes an each-all subrelation based on $\mathrm{Y}^{2}$ and $\mathrm{Z}^{2}$, namely $\mathrm{Y}^{2} \times \mathrm{Z}^{2}$.

Transformation of 1st-order linear $\mathrm{M} \uparrow$ quantifications to 2nd-order relational quantifications: Consider the definiendum of Barwise's (17),

$$
\mathrm{Q}_{1} x \Phi x,
$$

where $\mathrm{Q}_{1}$ is $\mathrm{M} \uparrow$. (22) says, roughly, that $\mathrm{Q}_{1}$ elements have the property $\Phi$. But this is equivalent to saying that there is a set $\mathrm{X}$ with $\mathrm{Q}_{1}$ elements, such that each element $x$ of $\mathrm{X}$ possesses the property $\Phi$. By applying the relational procedure described above (assigning $\mathrm{Q}_{1} x$ the rank 1 , associating it with a unary relational variable, $\mathrm{X}^{1}$, and a relational formula, $\mathrm{X} x$ ) we transform (22) to a sentence that expresses just this equivalence:

$$
\exists \mathrm{X}^{1}\left[\mathrm{Q}_{1} x \mathrm{X} x \& \forall x(\mathrm{X} x \rightarrow \Phi x)\right] .
$$

This relational quantification is the Barwise definiens of (17). Now, consider

$$
\mathrm{Q}_{1} x \mathrm{Q}_{2} y \Phi x y,
$$


where $\mathrm{Q}_{1}$ and $\mathrm{Q}_{2}$ are $\mathrm{M} \uparrow$. By applying the same procedure as above we transform (24) into an equivalent 2 nd-order relational formula, namely

$$
\exists \mathrm{X}^{1} \mathrm{Y}^{2}\left[\mathrm{Q}_{1} x \mathrm{X} x \& \forall x\left(\mathrm{X} x \rightarrow \mathrm{Q}_{2} y \mathrm{Y} x y\right) \& \forall x y(\mathrm{Y} x y \rightarrow \Phi x y)\right]
$$

(Note how Yxy traces the quantifier dependency of $\mathrm{Q}_{2} y$ in a way similar to Henkin's functional terms.) This formula states three conditions on $\mathrm{X}$, $\mathrm{Y}$ and $\Phi$, respectively - a quantifier condition on $\mathrm{X}$, a quantifier condition on $\mathrm{Y}$ relative to $\mathrm{X}$, and an each-all condition on $\Phi$ relative to $\mathrm{Y}$. We will treat (25) as the definiens of (24). To see how a quantifier dependence of depth larger than 1 is reflected in the relational; definiens, note:

$$
\begin{aligned}
& \mathrm{Q}_{1} x \mathrm{Q}_{2} y \mathrm{Q}_{3} z \Phi x y z \\
&={ }_{\mathrm{Df}} \exists \mathrm{X}^{1} \mathrm{Y}^{2} \mathrm{Z}^{3}\left[\mathrm{Q}_{1} x \mathrm{X} x \& \forall x\left(\mathrm{X} x \rightarrow \mathrm{Q}_{2} y \mathrm{Y} x y\right)\right. \\
&\left.\quad \& \forall x y\left(\mathrm{Y} x y \rightarrow \mathrm{Q}_{3} z \mathrm{Z} x y z\right) \& \forall x y z(\mathrm{Z} x y z \rightarrow \Phi x y z)\right] .
\end{aligned}
$$

Motivation for Barwise's definition of the basic case of a genuine PM $\uparrow$ : Consider<smiles></smiles>

where $\mathrm{Q}_{1}$ and $\mathrm{Q}_{2}$ are any $\mathrm{M} \uparrow$ quantifiers. By applying the above procedure we arrive at the definiens of Barwise's (18): $\exists \mathrm{X}^{1} \mathrm{Y}^{1}\left[\mathrm{Q}_{1} x \mathrm{X} x\right.$ \& $\left.\mathrm{Q}_{2} y \mathrm{Y} y \& \forall x y(\mathrm{X} x \& \mathrm{Y} y \rightarrow \Phi x y)\right]$. Clearly, (18) is equivalent to Henkin's definition when $\mathrm{Q}_{1}$ and $\mathrm{Q}_{2}$ are standard quantifiers. Observe that situations in which (18) comes out true involve the same kind of "massive nucleus" as those in which Henkin's (1) is true. Interpreting $\mathrm{Q}_{1}$ and $\mathrm{Q}_{2}$ as "Most", a typical situation is:

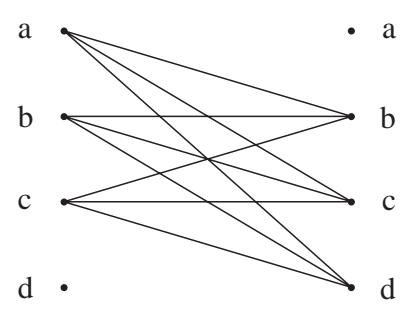

Graph II 
Examining the definiens of (18) we notice its similarity to (25): the first two conjuncts in the scope of the relational existential quantifiers express quantifier conditions on $\mathrm{X}$ and $\mathrm{Y}$. The last conjunct is an each-all condition on $\mathrm{X}$ and $\mathrm{Y}$ with respect to $\Phi$ or, more generally, an inclusion condition on $\langle\mathrm{X}, \mathrm{Y}\rangle$ in $\Phi$. Based on this analysis, I propose that we extend Barwise's definition of the basic cases of PM $\uparrow \mathrm{Q}$ to a general definition in the following way:

(Syn-2)

The syntax of PM $\uparrow$ Q (Syn-2) is obtained from the syntax of PSQ (Syn-1) by making the following changes:

A. In the definition of quantifier-prefix, $Q$, we replace (ii) by

(ii) $^{*}$ for $1 \leqslant i \leqslant n$, there is a $\mathrm{M} \uparrow$ generalized quantifier $\mathrm{Q}_{i}$ and a variable $x_{i}$ such that $q_{i}=\mathrm{Q}_{i} x_{i}$.

B. The definition of notions relative to $Q$ is now as follows:

1. $q_{i}$ is a quantifier atom.

2. If $q_{i}>q_{j}$ we say that $q_{i}$ governs $q_{j}$ and $q_{j}$ is dependent on $q_{i}$, and if there is no $q_{k}$ such that $q_{i}>q_{k}>q_{j}$, we say that $q_{i}$ is an immediate governor of $q_{j}$ and $q_{j}$ is an immediate dependent of $q_{i}$.

3. If $q_{i}$ has no dependents, we say that $q_{i}$ is a smallest quantifier atom. If $q_{i}$ has no governors, $q_{i}$ is said to be a largest quantifier atom.

4. The rank of $q_{i}, r\left(q_{i}\right)$, or simply $r_{i}$, is the number governors of $q_{i}+1$.

C. The method of syntactic representation is as follows: If $q_{i}>q_{j}$, then $q_{i}$ is to the left of $q_{j}$. If $q_{i}$ is an immediate governor of $q_{j}$, a line connects $q_{i}$ to $q_{j}$. If $q_{i}$ is a smallest quantifier atom, a line connects $q_{i}$ to the quantified formula.

(Sem-2)

The semantics of PM $\uparrow \mathrm{Q}$ (Sem-2) is obtained from the semantics of PSQ (Sem-1) by:

A. Adding all the (1st-order) $\mathrm{M} \uparrow$ quantifiers of $\mathrm{L}$ to the logical vocabulary of $\mathrm{L}^{\prime}$ (as before, $\mathrm{L}^{\prime}$ includes only the linear part of $\mathrm{L}$ ).

B. Replacing the transformation procedure for PSQs by:

Semantic Definition of $\mathrm{PM} \uparrow \mathrm{Q}$ (in the form of a procedure for transforming an arbitrary $\mathrm{PM} \uparrow \mathrm{Q}$ of $\mathrm{L}$ into a (linear) quantification of $\left.\mathrm{L}^{\prime}\right)$. Let 
$Q=\left(\left\{q_{1}, \ldots, q_{n}\right\},>\right)$ be a quantifier-prefix. With each quantifier atom $q_{i}$ we associate

(a) a distinct relational variable of $\mathrm{L}^{\prime}$ of arity $r_{i}$ (the rank of $q_{i}$ ),

$$
\mathrm{X}_{i}^{r_{i}}
$$

(b) a relational formula of $\mathrm{L}^{\prime}$,

$$
\mathrm{R}_{i}=\mathrm{X}_{i}^{r_{i}}\left(x_{i_{1}}, \ldots, x_{i_{\left(r_{i}\right)-1}}, x_{i}\right),
$$

where $q_{i_{1}}, \ldots, q_{i_{\left(r_{i}\right)-1}}$ are all the governors of $q_{i}$. (The order of variables in $\mathrm{R}_{i}$ does not matter, but in all its occurrences $\mathrm{R}_{i}$ has to present the same ordering of variables.) Note, again, how the individual variables in $\mathrm{R}_{i}$ trace the dependencies of $q_{i}$.

Let $\Phi$ be a wff of L. Then:

$(\mathrm{PM} \uparrow \mathrm{Q}) \quad Q \Phi={ }_{\mathrm{Df}} \exists \mathrm{X}_{1}^{r_{1}} \cdots \mathrm{X}_{n}^{r_{n}}\left(\mathrm{C}_{1} \& \cdots \& \mathrm{C}_{n} \& \mathrm{IN}\right)$.

Intuitively, $\mathrm{C}_{1}, \ldots, \mathrm{C}_{n}$ express the quantifier conditions set by the quantifier atoms of $Q$ and IN is the inclusion condition. For each quantifier atom $q_{i}, 1 \leqslant i \leqslant n, \mathrm{C}_{i}$ states the absolute or relative quantifier-condition expressed by $q_{i}$ : if $q_{i}$ is a largest quantifier atom, $\mathrm{C}_{i}$ expresses an absolute condition; otherwise, $\mathrm{C}_{i}$ expresses a condition relative to the immediate governors of $q_{i}$. IN relates the smallest quantifier atoms of $Q$ to the quantified formula, $\Phi$. Formally, we define $\mathrm{C}_{1}, \ldots, \mathrm{C}_{n}$ and IN as follows: For $1 \leqslant i \leqslant n$,

$$
\mathrm{C}_{i}= \begin{cases}\mathrm{Q}_{i} x_{i} \mathrm{R}_{i} & \text { if } r_{i}=1 \\ \forall \bar{x}_{j}\left(\mathrm{R}_{j_{1}} \& \cdots \& \mathrm{R}_{j_{k}} \rightarrow \mathrm{Q}_{i} x_{i} \mathrm{R}_{i}\right) & \text { otherwise, }\end{cases}
$$

where $\mathbf{R}_{j_{1}}, \ldots, \mathbf{R}_{j_{k}}$ are the relational formulas associated with all the immediate governors of $q_{i}$ and $\bar{x}_{j}$ is a sequence of all the individual variables in $\mathrm{R}_{j_{1}}, \ldots, \mathrm{R}_{j_{k}}$.

$$
\mathrm{IN}=\forall \bar{x}_{j}\left(\mathrm{R}_{j_{1}} \& \cdots \& \mathrm{R}_{j_{k}} \rightarrow \Phi\right)
$$

where $\mathbf{R}_{j_{1}}, \ldots, \mathbf{R}_{j_{k}}$ are the relational formulas associated with all the smallest quantifier atoms in $Q$ and $\bar{x}_{j}$ is defined as above. (Note that in IN $\bar{x}_{j}$ includes all the variables in $Q$.)

Examples. Clearly (17), (18), (21), (25) and (26) are all particular instances of the definition schema (PM $\uparrow$ Q). It might be worthwhile seeing how the definition works in somewhat more complicated cases. (In all the examples below the quantifiers are $\mathbf{M} \uparrow$.) 
(28)
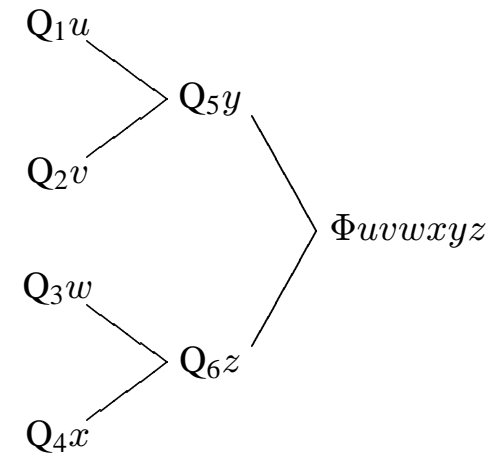

$={ }_{\mathrm{Df}} \exists \mathrm{U}^{1} \mathrm{~V}^{1} \mathrm{~W}^{1} \mathrm{X}^{1} \mathrm{Y}^{3} \mathrm{Z}^{3}\left[\mathrm{Q}_{1} u \mathrm{U} u \& \mathrm{Q}_{2} v \mathrm{~V} v \& \mathrm{Q}_{3} w \mathrm{~W} w\right.$ $\& \mathrm{Q}_{4} x \mathrm{X} x \& \forall u v\left(\mathrm{U} u \& \mathrm{~V} v \rightarrow \mathrm{Q}_{5} y \mathrm{Y} u v y\right)$

$\& \forall w x\left(\mathrm{~W} w \& \mathrm{X} x \rightarrow \mathrm{Q}_{6} z \mathrm{Z} w x z\right)$

$\& \forall u v w x y z($ Yuvy \& Zwxz $\rightarrow$ Фuvwxyz)];

(29)

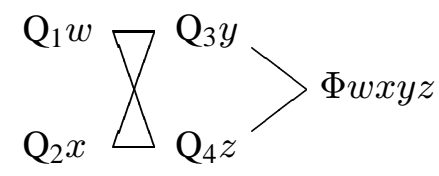

$={ }_{\text {Df }} \exists \mathrm{W}^{1} \mathrm{X}^{1} \mathrm{Y}^{3} \mathrm{Z}^{3}\left[\mathrm{Q}_{1} w \mathrm{~W} w \& \mathrm{Q}_{2} x \mathrm{X} x\right.$

$\& \forall w x\left(\mathrm{~W} w \& \mathrm{X} x \rightarrow \mathrm{Q}_{3} y \mathrm{Y} w x y\right)$

$\& \forall w x\left(\mathrm{~W} w \& \mathrm{X} x \rightarrow \mathrm{Q}_{4} z \mathrm{Z} w x z\right)$

$\& \forall w x y z(\mathrm{Y} w x y \& \mathrm{Z} w x z \rightarrow \Phi w x y z)] ;^{8}$

(30)

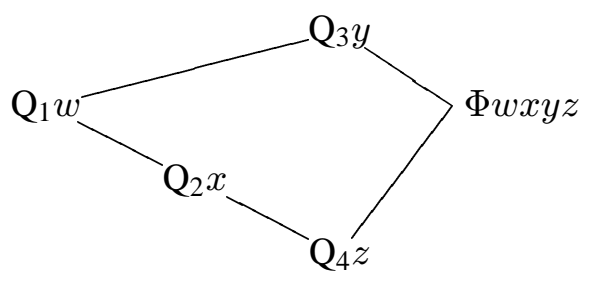

$$
\begin{aligned}
=_{\mathrm{Df}} & \exists \mathrm{W}^{1} \mathrm{X}^{2} \mathrm{Y}^{2} \mathrm{Z}^{3}\left[\mathrm{Q}_{1} w \mathrm{~W} w \& \forall w\left(\mathrm{~W} w \rightarrow \mathrm{Q}_{2} x \mathrm{X} w x\right)\right. \\
& \& \forall w\left(\mathrm{~W} w \rightarrow \mathrm{Q}_{3} y \mathrm{Y} w y\right) \\
& \& \forall w x\left(\mathrm{X} w x \rightarrow \mathrm{Q}_{4} z \mathrm{Z} w x z\right) \\
& \& \forall w x y z(\mathrm{Y} w y \& \mathrm{Z} w x z \rightarrow \Phi w x y z)] .
\end{aligned}
$$

Finally, the $\mathrm{PM} \uparrow \mathrm{Q}$ definiens of Henkin's (13) is:

$\exists \mathrm{P}^{1} \mathrm{~S}^{1} \mathrm{~T}^{1} \mathrm{U}^{1} \mathrm{~V}^{3} \mathrm{~W}^{3} \mathrm{X}^{4} \mathrm{Y}^{4} Z^{9}[\forall p \mathrm{P} p \& \forall s \mathrm{~S} s \& \forall t \mathrm{~T} t \& \forall u \mathrm{U} u$ $\& \forall p s(\mathrm{P} p \& \mathrm{~S} s \rightarrow \exists v \mathrm{~V} p s v) \& \forall t u(\mathrm{~T} t \& \mathrm{U} u \rightarrow \exists w \mathrm{~W} t u w)$ 


$$
\begin{aligned}
& \& \forall p s v(\mathrm{~V} p s v \rightarrow \forall x \mathrm{X} p s v x) \& \forall t u w(\mathrm{~W} t u w \rightarrow \forall y \mathrm{Y} t u w y) \\
& \& \forall p s t u v w x y(\mathrm{Xpsvx} \& \mathrm{Y} t u w y \rightarrow \exists z \mathrm{Z} p s t u v w x y z) \\
& \& \forall p s t u v w x y z(\mathrm{Z} p s t u v w x y z \rightarrow \Phi p s t u v w x y z)] .
\end{aligned}
$$

It is easy to check that (31) is equivalent to (14), assuming the axiom of choice.

Linguistic Applications. An example of an English PM $\uparrow \mathrm{Q}$ that is more complex than Barwise's (18) is the following:

Most actors in the studio have at least three fans and four critics who disagree about them.

Restricting the universe to actors in the studio, we can symbolize (32) by

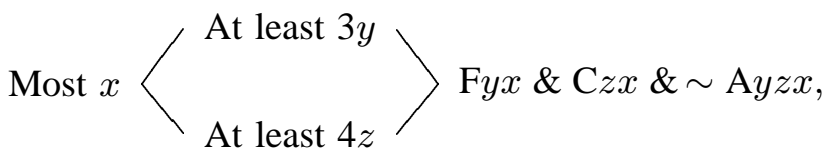

with the obvious interpretations of "F", "C" and "A". (33) is defined by

$$
\begin{aligned}
& \exists \mathrm{X}^{1} \mathrm{Y}^{2} \mathrm{Z}^{2}[\text { Most } x \mathrm{X} x \& \forall x(\mathrm{X} x \rightarrow \text { at least } 3 y \mathrm{Y} x y) \\
& \quad \& \forall x(\mathrm{X} x \rightarrow \text { at least } 4 z \mathrm{Z} x z) \\
& \quad \& \forall x y z(\mathrm{Y} x y \& \mathrm{Z} x z \rightarrow \mathrm{F} y x \& \mathrm{C} z x \& \mathrm{~A} y z x)] .
\end{aligned}
$$

(I specifically chose a sentence in which all the bound variables are restricted to objects with the same property - namely, being an actor in the studio - in order to make do with unary instead of binary quantifiers. As I indicated above, usually many-place quantifiers are required for the analysis of natural-language quantifications.)

We now arrive at the last step in the construction of a general definition of partially-ordered generalized quantifiers: the extension of (PM $\uparrow \mathrm{Q})$ to all generalized quantifiers, regardless of monotonicity.

\section{A GENERAL DEFINITION OF PGQ}

In "Branching Quantifiers in English" Barwise demonstrated that his definitions of the basic cases of $\mathrm{PM} \uparrow \mathrm{Q}$ fail to give reasonable results when the quantifiers involved are $\mathbf{M} \downarrow$, and clearly these definitions also fail when the quantifiers involved are non-M or mixed. ( $\mathrm{Q}$ is $\mathrm{M} \downarrow$ iff for any wffs $\Phi$ and $\Psi$, "Q $x \Phi x \& \forall x(\Psi x \rightarrow \Phi x)$ " implies " $\mathrm{Q} x \Psi x$ ". 
$\mathrm{Q}$ is non- $\mathrm{M}$ iff $\mathrm{Q}$ is neither $\mathrm{M} \uparrow$ nor $\mathrm{M} \downarrow$. "Less than $n$ ", "At most $n$ ", "Few", and "No" are $\mathrm{M} \downarrow$. "Exactly $n$ ", "Between $n$ and $m$ ", and "An even number of" are non-M.) Thus, consider

(35) There are at most two planets in our solar system.

Under Barwise's (17) this sentence is logically true since the empty set satisfies the definiens. But (35) is not even true, let alone logically true. So (17) fails to produce a general definition of " $\mathrm{Q} x \Phi x$ ". The situation is not better with respect to (18). When $\mathrm{Q}_{1}$ and $\mathrm{Q}_{2}$ are $\mathrm{M} \downarrow$, (18) turns out logically true (let $\mathrm{X}$ and $\mathrm{Y}$ be the empty set), but clearly not all $\mathrm{M} \downarrow$ quantifications of the form (18) are logically true. Since (17) and (18) are particular instances of the general definition of $\mathrm{PM} \uparrow \mathrm{Q}$, this definition cannot be considered a general definition of PGQ. Can we transcend the limitations of (PM $\uparrow \mathrm{Q})$ ?

In his 1979 paper Barwise gave a partially positive answer to this question. The meaning of branching $\mathbf{M} \uparrow$ quantifications, Barwise suggested, is different from that of branching $\mathrm{M} \downarrow$ quantifications. And a definition that captures the intuitive meaning of $\mathbf{M} \downarrow$ quantification will have the following basic clauses (pp. 63-64):

$$
\mathrm{Q}_{1} x \Phi x={ }_{\mathrm{Df}} \exists \mathrm{X}^{1}\left[\mathrm{Q}_{1} x \mathrm{X} x \& \forall x(\Phi x \rightarrow \mathrm{X} x)\right] ;
$$

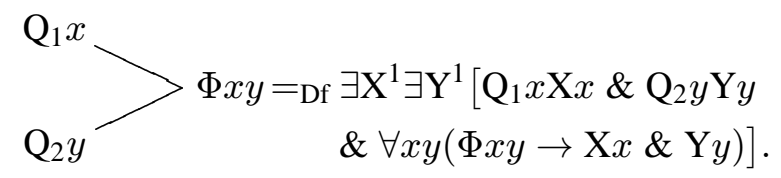

These definitions provide a reasonable interpretation of (35) as well as

Few linguists and few philosophers agree with each other about branching quantification,

and

(39) Nobody loves nobody,

understood in its colloquial sense (i.e., as meaning "Nobody loves anybody"). Barwise did not mention branching non-M quantifications, but with regard to mixed branching quantifications, Barwise struck a skeptical note: "There is no sensible way to interpret [branching quantifications] when one [quantifier] is increasing and the other decreasing. Thus, for example,

Few of the boys in my class and most of the girls in your class have all dated each other, 
appears grammatical, but it makes no sense." (Barwise 1979, pp. 65-66.)

In "Ways of Branching Quantifiers" I argued that Barwise's solution to the problem of extending his definition of (the basic cases of) $\mathrm{PM} \uparrow \mathrm{Q}$ to PGQ is inadequate. Barwise suggested that the meaning - and meaningfulness - of PGQ's is essentially connected with the monotonic features of the quantifiers involved, but general methodological considerations suggest otherwise. In particular, his claim is refuted by the case of linear generalized quantification, where quantifiers vary with respect to monotonicity as much as in non-linear quantification, yet the same semantic definition applies to all quantifiers (and all combinations of quantifiers) regardless of monotonicity. Since linear quantification falls under PGQ, PGQ is prima facie insensitive to monotonicity. Moreover, a closer look at Barwise's definition of a genuine PM $\downarrow Q$ shows that, unlike his definition of the basic case of $\mathrm{PM} \uparrow \mathrm{Q}$, the former is essentially a linear 1st-order quantification. Thus, what (37) says (when $\mathrm{Q}_{1}$ and $\mathrm{Q}_{2}$ are $\mathrm{M} \downarrow$ ) is that the domain of $\Phi$ contains $\mathrm{Q}_{1}$ elements and its range contains $\mathrm{Q}_{2}$ elements. I.e., assuming $\mathrm{Q}_{1}, \mathrm{Q}_{2}$ are $\mathrm{M} \downarrow$, the definiens of (37) is equivalent to:

$$
\mathrm{Q}_{1} x \exists y \Phi x y \& \mathrm{Q}_{2} y \exists x \Phi x y .
$$

But (41) makes sense - the same sense - for any sentence of the form (27), no matter what monotonicity features $\mathrm{Q}_{1}$ and $\mathrm{Q}_{2}$ exhibit. In "Ways of Branching Quantifiers" I suggested that there is more than one nonlinear form of quantification but each such form is universally applicable. The simplest kind of non-linear quantification is given by (41): " $\mathrm{Q}_{1} x$ and $\mathrm{Q}_{2} y$ stand in the relation $\mathrm{R}$ " means "There are $\mathrm{Q}_{1}$ things in $\operatorname{Dom}(\mathrm{R})$ and $\mathrm{Q}_{2}$ things in $\mathrm{Rng}(\mathrm{R})$ ". I called this branching structure independent quantification. (For further discussion see May, 1989; van Benthem, 1989.) Thus, on my interpretation, (38) and (39) exemplify an instance of independent quantification. But the universal structure discovered by Henkin is essentially more complex. Barwise showed how to extend it to one type of PGQ - PM $\uparrow$ Q it would take an extra step to extend it to PGQ's in general.

In what way should Barwise's definition be extended to a general definition of PGQ? In the literature, there are two different ways of approaching this problem. My own approach in Sher (1990a/1991a) is based on the considerations described above: There is one general notion, hence one general definition, of PGQ, regardless of monotonicity, and according to this definition a sentence with $\mathrm{M} \uparrow$ quantifiers says essentially the same thing as a sentence with $\mathrm{M} \downarrow$ quantifiers or with non-M quantifiers. However, a general definition can often be simplified in special cases, where a particular condition is trivially satisfied. This condition 
can then be omitted and the result is a simpler definition for the special case. $\mathrm{M} \uparrow$ quantifiers constitute such a special case with respect to the general definition of PGQ. Some condition that is essential for other quantifiers is trivially satisfied in a purely $\mathrm{M} \uparrow$ prefix, and this condition

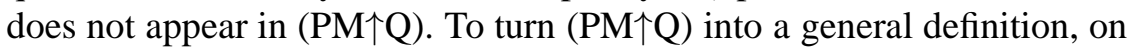
this approach, we have to find, and reinstate, the omitted condition. This condition, together with $\mathrm{C}_{1}, \ldots, \mathrm{C}_{n}$ and IN (see the general definition of $\mathrm{PM} \uparrow \mathrm{Q}$ above) will yield a universally applicable definition of PGQ.

An altogether different approach was proposed by Dag Westerståhl in "Branching Generalized Quantifiers and Natural Language" (1987). Westerståhl, too, believes there is a single definition of all PGQ's, but in his view this definition assigns different meanings to different statements according to the monotonicity properties of the quantifiers involved. (I.e., a $\mathbf{M} \uparrow$ quantification does say an intuitively different thing from a $\mathbf{M} \downarrow$ or a non-M quantification.) To give a general definition of PGQ we proceed in two steps. In step \#1 we give distinct definitions of partiallyordered quantifications based on monotonicity. In step \#2 we combine these partial definitions under a single umbrella, arriving at a general formula that applies to all combinations of quantifiers, irrespective of monotonicity.

Westerståhl found an ingenious method for combining three partial definitions of basic PGQ's into a single definition. The first two are Barwise's definitions of $\mathrm{M} \uparrow$ and $\mathrm{M} \downarrow$ branching quantifications. The third is a partial definition of non-M branching quantifiers due to Johan van Benthem (Westerståhl, 1987, pp. 269, 274): Let $\mathrm{Q}_{1}$ and $\mathrm{Q}_{2}$ be non-M quantifiers of the form "exactly $n$ ". Then,

$$
\mathrm{Q}_{1} x \Phi x={ }_{\mathrm{Df}} \exists \mathrm{X}^{1}\left[\mathrm{Q}_{1} x \mathrm{X} x \& \forall x(\Phi x \leftrightarrow \mathrm{X} x)\right] ;
$$

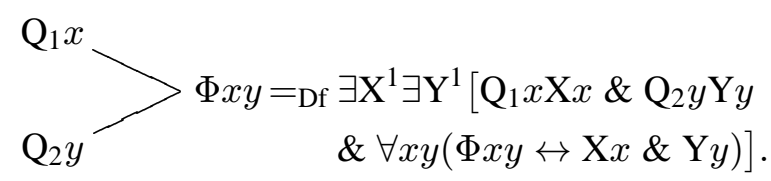

I will not describe Westerståhl's complex method here, but his method (at least as it stands in the 1987 paper) does not constitute a general definition of PGQ. Westerståhl's method is essentially limited in three ways (see Westerståhl, 1987; and Sher, 1990b): (A) It does not handle non-M quantifiers that (i) do not fall under van Benthem's category and (ii) are not definable (in a certain way) from $\mathrm{M} \uparrow$ and $\mathrm{M} \downarrow$ quantifiers. One such quantifier is "An even number of". (B) It is limited to homogeneous prefixes. I.e., it does not handle branching quantifications with mixed 
quantifiers (e.g., (40)). (C) Westerståhl provided definitions for several types of partially-ordered prefixes but did not show how to extend these definitions to prefixes of an arbitrary partial ordering. It is not clear whether his definitions can be extended to arbitrary orderings. Based on these limitations I conclude that Westerståhl's method, as it now stands, falls short of producing a general definition of PGQ.

The general definition PGQ I will present below is based on the earlier approach. Here we look for a single definition, applicable to all quantifiers, that can be simplified in the case of purely $\mathbf{M} \uparrow$ prefixes. The key to finding such a definition lies in linear quantification. Since the well understood form of linear generalized quantification falls under PGQ, it can be used both as a clue and as a test for a general definition of PGQ. My first step is to examine the 2nd-order definiens of the basic case of linear $\mathrm{M} \uparrow$ quantification under ( $\mathrm{PM} \uparrow \mathrm{Q}$ ) and search for an additional condition that, added to its definiens, yields a definition of a (basic) linear quantification in general regardless of monotonicity. Consider

$$
\mathrm{Q}_{1} x \Phi x .
$$

Assume $\mathrm{Q}_{1}$ is $\mathrm{M} \uparrow$. Then, according to Barwise's (17) the truth conditions of (22) are as follows: (22) is true in a model $\mathfrak{U}$ with a universe A iff there is a subset $\mathrm{X}$ of $\mathrm{A}$ such that:

(a) $\quad \mathrm{X}$ satisfies the quantifier condition $\mathrm{Q}_{1}$; and

(b) Each element of $X$ is in $\Phi^{\mathfrak{U}}$ (i.e., $X \subseteq \Phi^{\mathfrak{U}}$ ).

( $\Phi^{\mathfrak{U}}$ is the relation defined by $\Phi x$ in $\mathfrak{U}$.) Now, let us relax the requirement that $\mathrm{Q}_{1}$ is $\mathrm{M} \uparrow$. What condition should we add to (a) and (b) in order to obtain a correct definition of (22)? It is easy to see that a maximality condition on $\mathrm{X}$ will suffice:

(c) $\mathrm{X}$ is a maximal set satisfying (b).

I will call (c) the maximality condition on $\mathrm{X}$ with respect to (b). We can compress (b) and (c) into one condition:

(d) $\quad \mathrm{X}$ is a maximal set included in $\Phi^{\mathfrak{U}}$.

Formally, I extend Barwise's (17) as follows: Let $\mathrm{Q}_{1}$ be any quantifier; then

$$
\begin{aligned}
\mathrm{Q}_{1} x \Phi x={ }_{\mathrm{Df}} & \exists \mathrm{X}^{1}\left\{\mathrm{Q}_{1} x \mathrm{X} x \& \forall x(\mathrm{X} x \rightarrow \Phi x)\right. \\
& \& \forall \mathrm{X}^{\prime}\left[\forall x \left(\left(\mathrm{X} x \rightarrow \mathrm{X}^{\prime} x\right)\right.\right. \\
& \left.\left.\left.\&\left(\mathrm{X}^{\prime} x \rightarrow \Phi x\right)\right) \rightarrow \forall x\left(\mathrm{X}^{\prime} x \rightarrow \mathrm{X} x\right)\right]\right\} .
\end{aligned}
$$


Informally, we can express (44) as:

$$
\begin{gathered}
\mathrm{Q}_{1} x \Phi x={ }_{\mathrm{Df}} \exists \mathrm{X}^{1}\left[\mathrm{Q}_{1} x \mathrm{X} x \& \mathrm{X}\right. \text { is a maximal set such that } \\
\forall x(\mathrm{X} x \rightarrow \Phi x)] .
\end{gathered}
$$

It is easy to see that when $\mathrm{Q}$ in $\mathrm{M} \uparrow$, the maximality condition is redundant and (44) is logically equivalent to Barwise's (17). I.e., if Q is $\mathrm{M} \uparrow$, there is a set $\mathrm{X}$ satisfying (a) and (b) iff there is a set $\mathrm{X}^{*}$ satisfying (a), (b) and (c). In one direction, this is obvious. In the other direction: suppose $\mathrm{X}$ satisfies (a) and (b), and let $\mathrm{X}^{*}=\Phi$. Clearly $\mathrm{X}^{*}$ satisfies (b) and (c), and since $\mathrm{X}^{*} \supseteq \mathrm{X}$ and $\mathrm{Q}$ is $\mathrm{M} \uparrow, \mathrm{X}^{*}$ also satisfies (a). ${ }^{9}$

The above definition of (22) is a definition of the desired kind: it extends Barwise's (17) to all non-M $\uparrow$ quantifiers, and in the case of $\mathrm{M} \uparrow$ quantifiers, the extension is superfluous. The key to this definition is the maximality condition which is obvious in the present case but, as we shall now see, plays a crucial role in finding a general solution to the semantic problem of PGQ. Consider

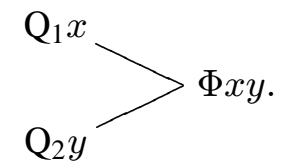

According to Barwise, when $\mathrm{Q}_{1}$ and $\mathrm{Q}_{2}$ are $\mathrm{M} \uparrow(27)$ is true in a model $\mathfrak{U}$ with a universe $\mathrm{A}$ iff there are two subsets, $\mathrm{X}$ and $\mathrm{Y}$, of $\mathrm{A}$ such that:

(a) $\quad \mathrm{X}$ satisfies $\mathrm{Q}_{1}$ and $\mathrm{Y}$ satisfies $\mathrm{Q}_{2}$; and

(b) $\quad$ For each $x$ in $\mathrm{X}$ and $y$ in $\mathrm{Y},\langle x, y\rangle$ is in $\Phi^{\mathfrak{U}}$

$$
\text { (i.e., } \mathrm{X} \times \mathrm{Y} \subseteq \Phi^{\mathfrak{U}} \text { ). }
$$

(a) includes quantifier conditions on $\mathrm{X}$ and $\mathrm{Y}$ and (b) is the each all (Cartesian product) or, more generally, inclusion condition on $\langle\mathrm{X}, \mathrm{Y}\rangle$ with respect to $\Phi$.

Now, to extend this definition of to all quantifiers, regardless of monotonicity, I suggest that we apply the same method as in (22) above. But while in the aforementioned case (b) involved a single set included in $\Phi^{\mathfrak{U}}$, here (b) involves a Cartesian product of two sets included in $\Phi^{\mathfrak{U}}$. I therefore formulate the maximality condition as follows:

(c) $\quad\langle\mathrm{X}, \mathrm{Y}\rangle$ is a maximal pair satisfying (b).

We can combine (b) and (c) into a single condition,

(d) $\quad\langle\mathrm{X}, \mathrm{Y}\rangle$ is maximal pair such that for each $x \in \mathrm{X}$ and each $y \in \mathrm{Y},\langle x, y\rangle \in \Phi^{\mathfrak{U}}$. 
I will call (d) the maximal inclusion (each-all) condition on $\langle\mathrm{X}, \mathrm{Y}\rangle$ with respect to $\Phi .^{10}$

Formally, I extend Barwise's (18) in the following way (see Sher, 1990a, pp. 412-413 / 1991a, p. 121). Let $\mathrm{Q}_{1}$ and $\mathrm{Q}_{2}$ be any quantifiers; then

$$
\begin{aligned}
\mathrm{Q}_{2} y & \Phi x y \\
= & \exists \mathrm{X}^{1} \mathrm{Y}^{1}\left\{\mathrm{Q}_{1} x \mathrm{X} x \& \mathrm{Q}_{2} y \mathrm{Y} y\right. \\
& \& \forall x y(\mathrm{X} x \& \mathrm{Y} y \rightarrow \Phi x y) \\
& \& \forall \mathrm{X}^{\prime} \mathrm{Y}^{\prime}\left[\forall x y \left(\left(\mathrm{X} x \& \mathrm{Y} y \rightarrow \mathrm{X}^{\prime} x \& \mathrm{Y}^{\prime} y\right)\right.\right. \\
& \left.\&\left(\mathrm{X}^{\prime} x \& \mathrm{Y}^{\prime} y \rightarrow \Phi x y\right)\right) \\
& \left.\left.\rightarrow \forall x y\left(\mathrm{X}^{\prime} x \& \mathrm{Y}^{\prime} y \rightarrow \mathrm{X} x \& \mathrm{Y} y\right)\right]\right\} .
\end{aligned}
$$

Informally:

$$
\begin{aligned}
\mathrm{Q}_{2} y & \mathrm{Q}_{1} x>\Phi x y={ }_{\mathrm{Df}} \exists \mathrm{X}^{1} \mathrm{Y}^{1}\left[\mathrm{Q}_{1} x \mathrm{X} x \& \mathrm{Q}_{2} y \mathrm{Y} y \&\langle\mathrm{X}, \mathrm{Y}\rangle\right. \\
& \text { is a max. pair s.t. } \\
& \forall x y(\mathrm{X} x \& \mathrm{Y} y \rightarrow \Phi x y)] .
\end{aligned}
$$

We can see that here, too, the maximality condition is redundant when $\mathrm{Q}_{1}$ and $\mathrm{Q}_{2}$ are $\mathrm{M} \uparrow$. I.e., if $\mathrm{Q}_{1}$ and $\mathrm{Q}_{2}$ are $\mathrm{M} \uparrow$, there is a pair $\langle\mathrm{X}, \mathrm{Y}\rangle$ satisfying (a) and (b) iff there is a pair $\left\langle\mathrm{X}^{*}, \mathrm{Y}^{*}\right\rangle$ satisfying (a), (b) and (c). In one direction this is obvious, in the other direction we could invoke Zorn's Lemma, but we do not have to. Without Zorn's Lemma we can prove the claim as follows: Assume $\mathrm{Q}_{1}, \mathrm{Q}_{2}$ are $\mathrm{M} \uparrow$ and suppose $\langle\mathrm{X}, \mathrm{Y}\rangle$ satisfies (a) and (b) in $\mathfrak{U}$. Let $\mathrm{X}^{*}=\{x \in \mathrm{A}: \forall y(\mathrm{Y} y \rightarrow \Phi x y)\}$. Clearly, $\mathrm{X}^{*} \supseteq \mathrm{X}$ and $\left\langle\mathrm{X}^{*}, \mathrm{Y}\right\rangle$ satisfies (b). Let $\mathrm{Y}^{*}=\left\{y \in \mathrm{A}: \forall x\left(\mathrm{X}^{*} x \rightarrow \Phi x y\right)\right\}$. Clearly, $\mathrm{Y}^{*} \supseteq \mathrm{Y}$ and $\left\langle\mathrm{X}^{*}, \mathrm{Y}^{*}\right\rangle$ satisfies (b). By construction, $\left\langle\mathrm{X}^{*}, \mathrm{Y}^{*}\right\rangle$ also satisfies (c). ${ }^{11}$ Now, $\mathrm{X}^{*} \supseteq \mathrm{X}, \mathrm{Y}^{*} \supseteq \mathrm{Y}$ and $\mathrm{Q}_{1}, \mathrm{Q}_{2}$ are $\mathrm{M} \uparrow$, hence $\left\langle\mathrm{X}^{*}, \mathrm{Y}^{*}\right\rangle$ satisfies (a). Q.E.D. (Remark: In the above proof the order of extending $\mathrm{X}$ and $\mathrm{Y}$ is immaterial. We may get a different pair if we extend $\mathrm{Y}$ before $\mathrm{X}$, but it will be a pair satisfying (a)-(c) all the same.)

Clearly, when $\mathrm{Q}_{1}$ and $\mathrm{Q}_{2}$ are $\mathrm{M} \downarrow$, the problem Barwise came upon when applying (18) to $\mathbf{\downarrow} \downarrow$ quantifiers does not arise: (27) comes out true only if there is a maximal Cartesian product included in $\Phi$ whose domain and range are small enough to satisfy $\mathrm{Q}_{1}$ and $\mathrm{Q}_{2}$. (When $\Phi$ is not empty, $\langle\phi, \phi\rangle-$ or for that matter, $\langle\mathrm{X}, \phi\rangle$ or $\langle\phi, \mathrm{Y}\rangle$, for any $\mathrm{X}$ 
and Y - fail to satisfy this condition.) Thus, when applied to the $M \downarrow$ branching quantification (39), (46) gives the right result: $\phi \times \phi$ is a maximal Cartesian product included in the relation " $x$ loves $y$ " iff " $x$ loves $y$ " is empty. ${ }^{12}$ (46) also gives a reasonable interpretation of non-M and mixed-M quantifications, e.g., Barwise's (40). Consider also:

A couple of boys in my class and a couple of girls in your class are all dating each other. [Sher 1990a, p. 414 / 1991a, p. 123.]

An even number of dots and an odd number of stars are all connected by lines.

An small number of dots and a large number of stars are all connected by lines.

Most of my friends have all applied to the same few graduate programs.

These sentences say that there is a group of $\mathrm{Q}_{1}$ members of a certain kind and a group of $\mathrm{Q}_{2}$ members of a certain kind, such that each member of the first group stands in a given relation to all the members of the second group. (The two groups create a maximal or massive nucleus of objects standing in the given relation. $)^{13}$ As in the case of (19) and (4), these examples involve binary generalized quantifiers, but the underlying principle is essentially captured by (46). For the binary version of (46) see Sher (1990a/1991a).

My present task is to create a general definition of PGQ - (PGQ) based on the principles exemplified in (44) and (46). Clearly, the quantifier conditions, $\mathrm{C}_{1}, \ldots, \mathrm{C}_{n}$, and the inclusion condition, $\mathrm{IN}$, will be as in (PM $\uparrow \mathrm{Q})$. The question to be settled is: How does the maximality condition extend to the general case? In addition to the requirement that (PGQ) capture the usual 1st-order truth conditions when applied to linear quantifier-prefixes, I will rely on the desideratum that (PGQ) coincide with (PM $\uparrow \mathrm{Q}$ ) when applied to $\mathrm{M} \uparrow$ prefixes. To show that the latter is satisfied I will indicate how an $n$-tuple of relations satisfying $\mathrm{C}_{1}, \ldots, \mathrm{C}_{n}$ and IN can be extended into a $n$-tuple of relations satisfying $\mathrm{C}_{1}, \ldots, \mathrm{C}_{n}$, IN and the maximality conditions. In order to simplify the notation, I will rewrite (44) and (46) in a new way, using the same form of expression for more complex quantifications:

$$
\begin{aligned}
\mathrm{Q}_{1} x \Phi x={ }_{\mathrm{Df}} & \exists \mathrm{X}^{1}\left[\mathrm{C}: \mathrm{Q}_{1} x \mathrm{X} x \& \mathrm{IN}: \forall x(\mathrm{X} x \rightarrow \Phi x)\right. \\
& \& \operatorname{MAX}(\mathrm{X} / \mathrm{IN}): \mathrm{X} \text { is a max. set satisfying } \mathrm{IN}] .
\end{aligned}
$$


(51)

$$
\begin{aligned}
\mathrm{Q}_{2} y & \Phi x y \\
= & \mathrm{Df}^{1} \mathrm{Y}^{1}\left[\mathrm{C}_{1}: \mathrm{Q}_{1} x \mathrm{X} x \& \mathrm{C}_{2}: \mathrm{Q}_{2} y \mathrm{Y} y\right. \\
& \& \mathrm{IN}: \forall x y(\mathrm{X} x \& \mathrm{Y} y \rightarrow \Phi x y) \\
& \& \mathrm{MAX}(\mathrm{X}, \mathrm{Y} / \mathrm{IN}):\langle\mathrm{X}, \mathrm{Y}\rangle \\
& \text { is a max. pair satisfying IN }] .
\end{aligned}
$$

We can immediately extend (51) to

$$
\begin{aligned}
& \longrightarrow x_{1} \cdots x_{n} \\
& ={ }_{\mathrm{Df}} \exists \mathrm{X}_{1}^{1} \cdots \mathrm{X}_{n}^{1}\left[\mathrm{C}_{1}: \mathrm{Q}_{1} x_{1} \mathrm{X}_{1} x_{1}\right. \\
& \& \cdots \& \mathrm{C}_{n}: \mathrm{Q}_{n} x_{n} \mathrm{X}_{n} x_{n} \\
& \& \mathrm{IN}: \forall x_{1} \cdots x_{n}\left(\mathrm{X}_{1} x_{1}\right. \\
& \left.\& \cdots \& \mathrm{X}_{n} x_{n} \rightarrow \Phi x_{1} \cdots x_{n}\right) \\
& \& \operatorname{MAX}\left(\mathrm{X}_{1}, \ldots, \mathrm{X}_{n} / \mathrm{IN}\right):\left\langle\mathrm{X}_{1}, \ldots, \mathrm{X}_{n}\right\rangle
\end{aligned}
$$

is a max. tuple satisfying IN].

When $\mathrm{Q}_{1}, \ldots, \mathrm{Q}_{n}$ are all $\mathrm{M} \uparrow$, we will extend an $n$-tuple, $\left\langle\mathrm{X}_{1}, \ldots, \mathrm{X}_{n}\right\rangle$ satisfying $\mathrm{C}_{1}-\mathrm{IN}$ into an $n$-tuple, $\left\langle\mathrm{X}_{1}^{*}, \ldots, \mathrm{X}_{n}^{*}\right\rangle$ satisfying $\mathrm{C}_{1}-\mathrm{MAX}\left(\mathrm{X}_{1}\right.$, $\left.\ldots, \mathrm{X}_{n} / \mathrm{IN}\right)$ as in (46). Again, the order of extending $\mathrm{X}_{1}, \ldots, \mathrm{X}_{n}$ does not matter. (We may obtain different tuples $\left\langle\mathrm{X}_{1}^{*}, \ldots, \mathrm{X}_{n}^{*}\right\rangle$, but if the sentence is true/false under one of these, it is true/false under all the others.)

Next, we would like to extend (44) to arbitrarily long linear quantifications. Consider, first, a 2-place linear quantification. It is clear that (53) is a correct definition while (54) is not.

$$
\begin{aligned}
\mathrm{Q}_{1} x \mathrm{Q}_{2} y & \Phi x y \\
={ }_{\mathrm{Df}} & \exists \mathrm{X}^{1} \mathrm{Y}^{2}\left[\mathrm{C}_{1}: \mathrm{Q}_{1} x \mathrm{X} x \& \mathrm{C}_{2}: \forall x\left(\mathrm{X} x \rightarrow \mathrm{Q}_{2} y \mathrm{Y} x y\right)\right. \\
& \& \mathrm{IN}: \forall x y(\mathrm{Y} x y \rightarrow \Phi x y) \\
& \& \operatorname{MAX}\left(\mathrm{X} / \mathrm{C}_{2}\right): \mathrm{X} \text { is a max. set satisfying } \mathrm{C}_{2} \\
& \& \operatorname{MAX}(\mathrm{Y} / \mathrm{IN}): \mathrm{Y} \text { is a max. relation satisfying IN }] .
\end{aligned}
$$

$$
\mathrm{Q}_{1} x \mathrm{Q}_{2} y \Phi x y
$$




$$
\begin{aligned}
=_{\mathrm{Df}} & \exists \mathrm{X}^{1} \mathrm{Y}^{2}\left[\mathrm{C}_{1}: \mathrm{Q}_{1} x \mathrm{X} x \& \mathrm{C}_{2}: \forall x\left(\mathrm{X} x \rightarrow \mathrm{Q}_{2} y \mathrm{Y} x y\right)\right. \\
& \& \mathrm{IN}: \forall x y(\mathrm{Y} x y \rightarrow \Phi x y) \\
& \& \operatorname{MAX}\left(\mathrm{X}, \mathrm{Y} / \mathrm{C}_{2}, \mathrm{IN}\right):\langle\mathrm{X}, \mathrm{Y}\rangle
\end{aligned}
$$

is a max. pair sat. both $\mathrm{C}_{2}$ and IN].

To see that (54) fails, let $\mathrm{Q}_{1}=\mathrm{Q}_{2}=$ "Exactly 1", and let $\Phi=\{\langle a, c\rangle$, $\langle a, d\rangle\}$. Then, on the usual 1 st-order analysis the definiendum is false, but the definiens of (54) comes out true. (Let $\mathrm{X}=\{a\}, \mathrm{Y}=\{\langle a, c\rangle\}$.) The fact that (53) rather than (54) is a correct definition of 2-place linear quantifications indicates that generally (PGQ) includes multiple maximality conditions. It is easy to see that in the case of linear quantifier-prefixes, each quantifier has its own maximality condition:

$$
\begin{aligned}
\mathrm{Q}_{1} x_{1} \cdots & \mathrm{Q}_{n} x_{n} \Phi x_{1} \cdots x_{n} \\
={ }_{\mathrm{Df}} & \exists \mathrm{X}_{1}^{1} \cdots \mathrm{X}_{n}^{n}\left[\mathrm{C}_{1}: \mathrm{Q}_{1} x_{1} \mathrm{X}_{1} x_{1}\right. \\
& \& \mathrm{C}_{2}: \forall x_{1}\left(\mathrm{X}_{1} x_{1} \rightarrow \mathrm{Q}_{2} x_{2} \mathrm{X}_{2} x_{1} x_{2}\right) \\
& \& \cdots \\
& \& \mathrm{C}_{n}: \forall x_{1} \cdots x_{n-1}\left(\mathrm{X}_{n-1} x_{1} \cdots x_{n-1}\right. \\
& \left.\rightarrow \mathrm{Q}_{n} x_{n} \mathrm{X}_{n} x_{1} \cdots x_{n}\right) \\
& \& \mathrm{IN}: \forall x_{1} \cdots x_{n}\left(\mathrm{X}_{n} x_{1} \cdots x_{n} \rightarrow \Phi x_{1} \cdots x_{n}\right) \\
& \& \operatorname{MAX}\left(\mathrm{X}_{1} / \mathrm{C}_{2}\right): \mathrm{X}_{1} \\
& \text { is a } \max . \text { set satisfying } \mathrm{C}_{2} \\
& \& \cdots \\
& \& \operatorname{MAX}\left(\mathrm{X}_{n-1} / \mathrm{C}_{n}\right): \mathrm{X}_{n-1} \\
& \text { is a } \max . \text { relation satisfying } \mathrm{C}_{n} \\
& \& \operatorname{MAX}\left(\mathrm{X}_{n} / \mathrm{IN}\right): \mathrm{X}_{n} \\
& \text { is a } \max . \text { relation satisfying IN }] .
\end{aligned}
$$

Now, suppose that (53) is a $\mathrm{M} \uparrow$ quantification, and let $\langle\mathrm{X}, \mathrm{Y}\rangle$ be a pair satisfying $\mathrm{C}_{1}-\mathrm{IN}$. We extend $\langle\mathrm{X}, \mathrm{Y}\rangle$ to a pair $\left\langle\mathrm{X}^{*}, \mathrm{Y}^{*}\right\rangle$ satisfying $\mathrm{C}_{1}-\mathrm{MAX}(\mathrm{Y} / \mathrm{IN})$ as follows: First we extend $\mathrm{Y}$ to a relation $\mathrm{Y}^{*}$ satisfying IN (clearly, $\mathrm{Y}^{*}=\Phi$ ). By construction, $\left\langle\mathrm{X}, \mathrm{Y}^{*}\right\rangle$ satisfies IN and $\operatorname{MAX}(\mathrm{Y} / \mathrm{IN}) .\left\langle\mathrm{X}, \mathrm{Y}^{*}\right\rangle$ also satisfies $\mathrm{C}_{1}$ (because $\mathrm{X}$ does) and $\mathrm{C}_{2}$ (because $\mathrm{Y} \subseteq \mathrm{Y}^{*}$ and $\mathrm{Q}_{2}$ is $\mathrm{M} \uparrow$ ). Next we extend $\mathrm{X}$ to a set $\mathrm{X}^{*}$ such that $\left\langle\mathrm{X}^{*}, \mathrm{Y}^{*}\right\rangle$ satisfies $\operatorname{MAX}\left(\mathrm{X} / \mathrm{C}_{2}\right) .\left\langle\mathrm{X}^{*}, \mathrm{Y}^{*}\right\rangle$ also satisfies $\mathrm{C}_{1}$ ( since $\mathrm{X} \subseteq \mathrm{X}^{*}$ and $\mathrm{Q}_{1}$ is $\mathrm{M} \uparrow$ ), $\mathrm{C}_{2}$ (by construction), IN and $\mathrm{MAX}(\mathrm{Y} / \mathrm{IN})$ (since $\mathrm{Y}^{*}$ does). This completes our demonstration that when $\mathrm{Q}_{1}$ and $\mathrm{Q}_{2}$ are $\mathrm{M} \uparrow$ the maximality conditions are redundant. For our procedure to be adequate, it is essential that we extend $\mathrm{Y}$ before we extend $\mathrm{X}$ since $\mathrm{MAX}(\mathrm{Y} / \mathrm{IN})$ 
is independent of $\operatorname{MAX}\left(\mathrm{X} / \mathrm{C}_{2}\right)$ but not the other way around. In other words, if we extend $\mathrm{Y}$ to a $\mathrm{Y}^{*}$ satisfying $\mathrm{MAX}(\mathrm{Y} / \mathrm{IN})$ and then $\mathrm{X}$ to an $\mathrm{X}^{*}$ satisfying $\operatorname{MAX}\left(\mathrm{X} / \mathrm{C}_{2}\right)$, we do not have to worry about the possibility that $\mathrm{Y}^{*}$ can be further extended. $\mathrm{Y}^{*}$ is not affected by changes in $\mathrm{X}$. But changes in $Y$ do affect the maximization of $X$. So, if we extend $X$ to $\mathrm{X}^{*}$ first and then $\mathrm{Y}$ to $\mathrm{Y}^{*}$, we will have to go back to $\mathrm{X}^{*}$ and extend it in order to satisfy $\operatorname{MAX}\left(\mathrm{X} / \mathrm{C}_{2}\right)$. As a rule, we always extend relations associated with smaller quantifiers before we extend relations associated with larger quantifiers.

Comment: The PGQ reading of linear quantifications is more natural when we view these quantifications from right to left rather than from left to right. Thus viewed, (53) makes the following assertion: Take the relation $\Phi$ (the maximal relation $\mathrm{Y}$ included in $\Phi$ ); consider the set $\mathrm{X}$ of all $x$ 's which stand in the relation $\Phi(=\mathrm{Y})$ to $\mathrm{Q}_{2}$ things in the universe (to $\mathrm{Q}_{2} y$ 's): this set has exactly $\mathrm{Q}_{1}$ elements. This right-to-left reading is obtained by changing the order of the conjuncts in the definiens. Note that on this right-to-left reading it becomes clear that the inner quantifier(s) define a certain complex predicate, while the outermost quantifier makes an assertion: such-and-such a number of objects in the universe satisfy the complex predicate defined by the inner quantifier(s).

Based on the foregoing analysis I arrive at the following account of the maximality requirement:

A. The maximality requirement involves all the quantifiers in a given quantifier-prefix.

B. The maximality requirement concerns the relation of quantifiers to their immediate dependent(s), the quantified formula being regarded as the immediate dependent of the smallest quantifier(s).

C. The maximality requirement is expressed by a series of conditions on the relations associated with the quantifiers in Q.

D. The maximality conditions apply to clusters of quantifiers (relations associated with quantifiers). The smallest quantifiers make up one cluster. In a linear ordering each quantifier constitutes it own cluster.

The idea that a maximality condition applies to a cluster of quantifiers has to be made definite. To make this idea definite we have to (a) define "a cluster", (b) determine how many maximality conditions are associated with each cluster. The main issues are these: (a) Intuitively, a cluster is a set of quantifiers that share their immediate dependents. But what happens when two quantifiers share some, but not all, of their immediate dependents? (b) In simple cases, a maximality condition relates a cluster of quantifiers to a single immediate dependent. But what happens when there is more than one immediate dependent? Do we have more than 
one maximality condition for the given cluster? (One for each immediate dependent?) I will begin with the second question. Consider

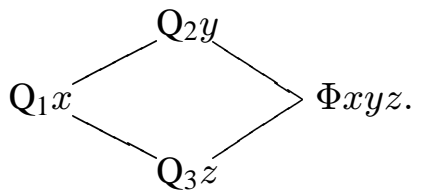

Here, $\left\{\mathrm{Q}_{1} x\right\}$ is a cluster, and $\mathrm{Q}_{1} x$ has two immediate dependents, $\mathrm{Q}_{2} y$ and $\mathrm{Q}_{3} z$. Should we assign one or two maximality conditions to $\left\{\mathrm{Q}_{1} x\right\}$ ? I.e., should we define (56) by

$$
\begin{aligned}
& \exists \mathrm{X}^{1} \mathrm{Y}^{2} \mathrm{Z}^{2}\left[\mathrm{C}_{1}: \mathrm{Q}_{1} x \mathrm{X} x \& \mathrm{C}_{2}: \forall x\left(\mathrm{X} x \rightarrow \mathrm{Q}_{2} y \mathrm{Y} x y\right)\right. \\
& \quad \& \mathrm{C}_{3}: \forall x\left(\mathrm{X} x \rightarrow \mathrm{Q}_{3} z \mathrm{Z} x z\right) \\
& \quad \& \mathrm{IN}: \forall x y z(\mathrm{Y} x y \& \mathrm{Z} x z \rightarrow \Phi x y z) \\
& \quad \& \operatorname{MAX}\left(\mathrm{X} / \mathrm{C}_{2}, \mathrm{C}_{3}\right): \mathrm{X} \text { is a max. set satisfying } \mathrm{C}_{2}, \mathrm{C}_{3} \\
& \quad \& \operatorname{MAX}(\mathrm{Y}, \mathrm{Z} / \mathrm{IN}):\langle\mathrm{Y}, \mathrm{Z}\rangle \text { is a max. pair satisfying IN]},
\end{aligned}
$$

or by

$$
\begin{aligned}
& \exists \mathrm{X}^{1} \mathrm{Y}^{2} \mathrm{Z}^{2}\left[\mathrm{C}_{1}: \mathrm{Q}_{1} x \mathrm{X} x \& \mathrm{C}_{2}: \forall x\left(\mathrm{X} x \rightarrow \mathrm{Q}_{2} y \mathrm{Y} x y\right)\right. \\
& \quad \& \mathrm{C}_{3}: \forall x\left(\mathrm{X} x \rightarrow \mathrm{Q}_{3} z \mathrm{Z} x z\right) \\
& \text { \& IN: } \forall x y z(\mathrm{Y} x y \& \mathrm{Z} x z \rightarrow \Phi x y z) \\
& \text { \& } \operatorname{MAX}\left(\mathrm{X} / \mathrm{C}_{2}\right): \mathrm{X} \text { is a max. set satisfying } \mathrm{C}_{2} \\
& \quad \& \operatorname{MAX}\left(\mathrm{X} / \mathrm{C}_{3}\right): \mathrm{X} \text { is a max. set satisfying } \mathrm{C}_{3} \\
& \quad \& \operatorname{MAX}(\mathrm{Y}, \mathrm{Z} / \mathrm{IN}):\langle\mathrm{Y}, \mathrm{Z}\rangle \text { is a max. pair satisfying IN }] ?
\end{aligned}
$$

To answer this question I turn to the requirement that (PGQ) be reducible to $(\mathrm{PM} \uparrow \mathrm{Q})$ when all the quantifiers involved are $\mathrm{M} \uparrow$. Now, suppose $\mathrm{Q}_{1}-$ $\mathrm{Q}_{3}$ are $\mathrm{M} \uparrow$ and $\langle\mathrm{X}, \mathrm{Y}, \mathrm{Z}\rangle$ satisfies $\mathrm{C}_{1}-\mathrm{IN}$. Then (57) complies with this requirement, but in (58) there can be a conflict between the two maximality conditions on $\mathrm{X}$. It is possible that no single extension of $\mathrm{X}$ satisfies both $\operatorname{MAX}\left(\mathrm{X} / \mathrm{C}_{2}\right)$ and $\operatorname{MAX}\left(\mathrm{X} / \mathrm{C}_{3}\right)$. Therefore, (57) is preferred. And in general, each cluster of quantifiers is assigned at most one maximality condition.

Now to the first question: When several quantifiers share some but not all of their immediate dependents, do they belong to the same cluster? Does each belong to a single cluster? A simple quantification exemplifying this situation is

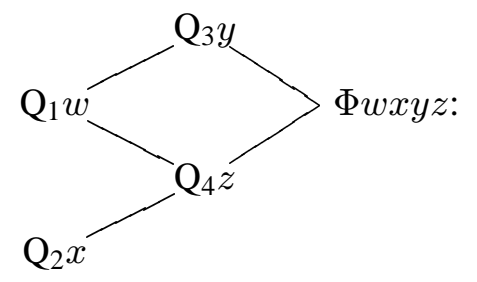


$\mathrm{Q}_{1} w$ shares immediate government over $\mathrm{Q}_{4} z$, but not over $\mathrm{Q}_{3} y$, with $\mathrm{Q}_{2} x$. The same considerations as above lead us to construct a single maximality condition for $\mathrm{Q}_{1} w$ and $\mathrm{Q}_{2} x$ with respect to all the immediate dependents of either one, i.e., with respect to both $\mathrm{Q}_{3} y$ and $\mathrm{Q}_{4} z$. We thus define (59) by:

$$
\begin{aligned}
& \exists \mathrm{W}^{1} \mathrm{X}^{1} \mathrm{Y}^{2} \mathrm{Z}^{3}\left[\mathrm{C}_{1}: \mathrm{Q}_{1} w \mathrm{~W} w \& \mathrm{C}_{2}: \mathrm{Q}_{2} x \mathrm{X} x\right. \\
& \quad \& \mathrm{C}_{3}: \forall w\left(\mathrm{~W} w \rightarrow \mathrm{Q}_{3} y \mathrm{Y} w y\right) \\
& \quad \& \mathrm{C}_{4}: \forall w x\left(\mathrm{~W} w \& \mathrm{X} x \rightarrow \mathrm{Q}_{4} z \mathrm{Z} w x z\right) \\
& \quad \& \mathrm{IN}: \forall w x y z(\mathrm{Y} w y \& \mathrm{Z} w x z \rightarrow \Phi w x y z) \\
& \quad \& \operatorname{MAX}\left(\mathrm{W}, \mathrm{X} / \mathrm{C}_{3}, \mathrm{C}_{4}\right):\langle\mathrm{W}, \mathrm{X}\rangle \\
& \text { is a max. pair satisfying } \mathrm{C}_{3}, \mathrm{C}_{4} \\
& \quad \& \operatorname{MAX}(\mathrm{Y}, \mathrm{Z} / \mathrm{IN}):\langle\mathrm{Y}, \mathrm{Z}\rangle \text { is a max. pair satisfying IN }] .
\end{aligned}
$$

Based on the above considerations I replace (D) by

E. The maximality conditions apply to clusters of quantifiers. A cluster of quantifiers is an equivalence class under the relation of common immediate government, defined as a transitive relation. Each cluster is subject to a single maximality condition. This maximality condition concerns all the quantifier-conditions associated with immediate dependents of quantifiers in the cluster (IN, when the cluster includes smallest quantifiers).

The particular cases discussed in (D) are not changed: the set of all smallest quantifiers is a cluster, and each cluster in a linear quantification is a singleton set.

(Syn-3)

The syntax of PGQ (Syn-3) is obtained from the syntax of PM $\uparrow$ (Syn-2) by:

A. Replacing (ii)* in the definition of quantifier-prefix by

(ii) $^{* *}$ for $1 \leqslant i \leqslant n$, there is a generalized quantifier $\mathrm{Q}_{i}$ and a variable $x_{i}$ such that $q_{i}=\mathrm{Q}_{i} x_{i}$.

B. Adding two new notions relative to $Q$ :

5. The relation of common immediate government, $\mathrm{G}$, is the smallest relation satisfying: (i) $q_{i} \mathrm{G} q_{i}$; (ii) if for some $q_{k}, q_{i}$ and $q_{j}$ are immediate governors of $q_{k}$, then $q_{i} \mathrm{G} q_{j}$; (iii) if $q_{i} \mathrm{G} q_{j}$ and $q_{j} \mathrm{G} q_{k}$, then $q_{i} \mathrm{G} q_{k}$; and (iv) if $q_{i}$ and $q_{j}$ are smallest quantifier atoms, then $q_{i} \mathrm{G} q_{j}$. 
6. A cluster of quantifiers, $C l$, is an equivalence class under the relation G. Clearly, there is exactly one cluster with smallest quantifier atoms. We will call it the smallest cluster.

(Sem-3)

The semantics of PGQ (Sem-3) is obtained from the semantics of PM $\uparrow \mathrm{Q}$ (Sem-2) by:

A. Adding all the (1st-order) generalized quantifiers of L to the logical vocabulary of $L^{\prime}$.

B. Replacing the passage beginning with "Let $\Phi$ be a wff of $L$ " in the Semantic Definition of $\mathrm{PM} \uparrow \mathrm{Q}$ by the following:

Let $\Phi$ be a wff of L. Let $C l_{1}, \ldots, C l_{m}$ be all the quantifier clusters in $Q$. Then:

$$
\text { (PGQ) } \quad \begin{aligned}
Q \Phi= & \\
\mathrm{Df} & \exists \mathrm{X}_{1}^{r_{1}} \cdots \mathrm{X}_{n}^{r_{n}}\left[\mathrm{C}_{1} \& \cdots \& \mathrm{C}_{n} \& \mathrm{IN} \&\right. \\
& \left.\& \operatorname{MAX}\left(C l_{1}\right) \& \cdots \& \operatorname{MAX}\left(C l_{m}\right)\right] .
\end{aligned}
$$

The intuitive explanation and formal definitions of $\mathrm{C}_{1}, \ldots, \mathrm{C}_{n}$ and IN are as in $(\mathrm{PM} \uparrow \mathrm{Q})$. $\mathrm{MAX}\left(C l_{1}\right), \ldots, \operatorname{MAX}\left(C l_{m}\right)$ are, intuitively, the maximality conditions on the clusters of quantifier atoms in $Q$ relative to their immediate dependents. Formally, $\operatorname{MAX}\left(C l_{1}\right), \ldots, \operatorname{MAX}\left(C l_{m}\right)$ are defined as follows: Let $C l_{i}=\left\{q_{i_{1}}, \ldots, q_{i_{j}}\right\}$ be a quantifier cluster, and let $q_{h_{1}}, \ldots, q_{h_{k}}$ be all the immediate dependents of quantifier atoms in $C l_{i}$, if any. Then:

$$
\begin{aligned}
\operatorname{MAX}( & \left.C l_{i}\right) \\
= & \forall \overline{\mathrm{X}}_{i}^{\prime}\left\{\forall \overline { x } _ { i } \left[\left(\mathrm{R}_{i_{1}} \& \cdots \& \mathrm{R}_{i_{j}} \rightarrow \mathrm{R}_{i_{1}}^{\prime} \& \cdots \& \mathrm{R}_{i_{j}}^{\prime}\right)\right.\right. \\
& \left.\& \Omega\left(\mathrm{X}_{i_{1}}^{\prime} / \mathrm{X}_{i_{1}}, \ldots, \mathrm{X}_{i_{j}}^{\prime} / \mathrm{X}_{i_{j}}\right)\right] \\
& \left.\rightarrow \forall \bar{x}_{i}\left(\mathrm{R}_{i_{1}}^{\prime} \& \cdots \& \mathrm{R}_{i_{j}}^{\prime} \rightarrow \mathrm{R}_{i_{1}} \& \cdots \& \mathrm{R}_{i_{j}}\right)\right\}
\end{aligned}
$$

where

(a) $\mathrm{R}_{i_{1}}^{\prime}, \ldots, \mathrm{R}_{i_{j}}^{\prime}$ are obtained from $\mathrm{R}_{i_{1}}, \ldots, \mathrm{R}_{i_{j}}$ by replacing the relational variables $\mathrm{X}_{i_{1}}, \ldots, \mathrm{X}_{i_{j}}$ by new relational variables, $\mathrm{X}_{i_{1}}^{\prime}, \ldots, \mathrm{X}_{i_{j}}^{\prime}$, of the same arity, respectively;

(b) $\bar{X}_{i}^{\prime}$ is any permutation of $\left\langle\mathrm{X}_{i_{1}}^{\prime}, \ldots, \mathrm{X}_{i_{j}}^{\prime}\right\rangle$;

(c) $\Omega=$ IN if $C l_{i}$ is the smallest cluster; otherwise, $\Omega=\mathrm{C}_{h_{1}} \& \cdots$ $\& \mathrm{C}_{h_{k}}$

(d) $\Omega\left(\mathrm{X}_{i_{1}}^{\prime} / \mathrm{X}_{i_{1}}, \ldots, \mathrm{X}_{i_{j}}^{\prime} / \mathrm{X}_{i_{j}}\right)$ is the result of replacing all occurrences of $\mathrm{X}_{i_{1}}$ by $\mathrm{X}_{i_{1}}^{\prime}, \ldots, \mathrm{X}_{i_{j}}$ by $\mathrm{X}_{i_{j}}^{\prime}$ in $\Omega$. 
Examples. Clearly (44), (46), (52), (53), (55), (57), (60) are instances of (PGQ). I will show how (1), (28), (29), (30) are defined when their quantifiers are not restricted.

(61)

$$
\begin{aligned}
\mathrm{Q}_{1} w-\mathrm{Q}_{3} y & \mathrm{Q}_{4} z \\
\mathrm{Q}_{\mathrm{Df}} & \exists \mathrm{W}^{1} \mathrm{X}^{1} \mathrm{Y}^{2} \mathrm{Z}^{2}\left[\mathrm{C}_{1}: \mathrm{Q}_{1} w \mathrm{~W} w \& \mathrm{C}_{2}: \mathrm{Q}_{2} x \mathrm{X} x\right. \\
\& \mathrm{C}_{3}: & \forall w\left(\mathrm{~W} w \rightarrow \mathrm{Q}_{3} y \mathrm{Y} w y\right) \\
\& \mathrm{C}_{4}: & \forall x\left(\mathrm{X} x \rightarrow \mathrm{Q}_{4} z \mathrm{Z} x z\right) \\
\& \mathrm{IN}: & \forall w x y z(\mathrm{Y} w y \& \mathrm{Z} x z \rightarrow \Phi w x y z)
\end{aligned}
$$

$\& \operatorname{MAX}\left(\mathrm{W} / \mathrm{C}_{3}\right)$ : $\mathrm{W}$ is a max. set satisfying $\mathrm{C}_{3}$ $\& \operatorname{MAX}\left(\mathrm{X} / \mathrm{C}_{4}\right): \mathrm{X}$ is a max. set satisfying $\mathrm{C}_{4}$ $\& \operatorname{MAX}(\mathrm{Y}, \mathrm{Z} / \mathrm{IN}):\langle\mathrm{Y}, \mathrm{Z}\rangle$

is a max. pair satisfying $\mathrm{IN}] .^{14}$

(62)
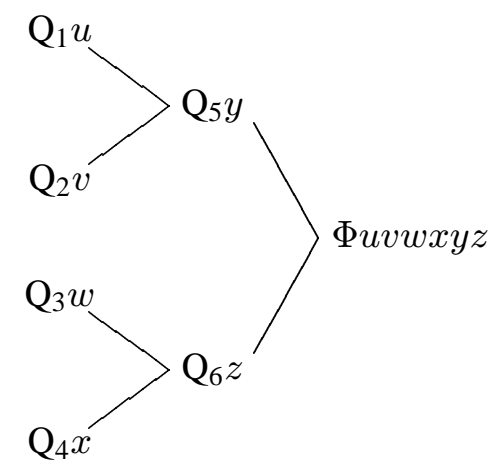

$={ }_{\mathrm{Df}} \exists \mathrm{U}^{1} \mathrm{~V}^{1} \mathrm{~W}^{1} \mathrm{X}^{1} \mathrm{Y}^{3} \mathrm{Z}^{3}\left[\mathrm{C}_{1}: \mathrm{Q}_{1} u \mathrm{U} u \& \mathrm{C}_{2}: \mathrm{Q}_{2} v \mathrm{~V} v\right.$

$\& \mathrm{C}_{3}: \mathrm{Q}_{3} w \mathrm{~W} w \& \mathrm{C}_{4}: \mathrm{Q}_{4} x \mathrm{X} x$

$\& \mathrm{C}_{5}: \forall u v\left(\mathrm{U} u \& \mathrm{~V} v \rightarrow \mathrm{Q}_{5} y \mathrm{Yuvy}\right)$

$\& \mathrm{C}_{6}: \forall w x\left(\mathrm{~W} w \& \mathrm{X} x \rightarrow \mathrm{Q}_{6} z \mathrm{Z} w x z\right)$

\& IN: $\forall u v w x y z($ Yuvy \& Zwxz $\rightarrow$ Фuvwxyz)

$\& \operatorname{MAX}\left(\mathrm{U}, \mathrm{V} / \mathrm{C}_{5}\right):\langle\mathrm{U}, \mathrm{V}\rangle$

is a max. pair satisfying $\mathrm{C}_{5}$

$\& \operatorname{MAX}\left(\mathrm{W}, \mathrm{X} / \mathrm{C}_{6}\right):\langle\mathrm{W}, \mathrm{X}\rangle$

is a max. pair satisfying $\mathrm{C}_{6}$

$\& \operatorname{MAX}(\mathrm{Y}, \mathrm{Z} / \mathrm{IN}):\langle\mathrm{Y}, \mathrm{Z}\rangle$

is a max. pair satisfying $\mathrm{IN}]$. 
(63)

$$
\begin{aligned}
\mathrm{Q}_{2} x & \mathrm{Q}_{1} w \\
=\mathrm{Q}_{4} z & \exists \mathrm{W}^{1} \mathrm{X}^{1} \mathrm{Y}^{3} \mathrm{Z}^{3}\left[\mathrm{C}_{1}: \mathrm{Q}_{1} w \mathrm{~W} w \& \mathrm{C}_{2}: \mathrm{Q}_{2} x \mathrm{X} x\right. \\
& \& \mathrm{C}_{3}: \forall w x\left(\mathrm{~W} w \& \mathrm{X} x \rightarrow \mathrm{Q}_{3} y \mathrm{Y} w x y\right) \\
& \& \mathrm{C}_{4}: \forall w x\left(\mathrm{~W} w \& \mathrm{X} x \rightarrow \mathrm{Q}_{4} z \mathrm{Z} w x z\right) \\
& \& \mathrm{IN}: \forall w x y z(\mathrm{Y} w x y \& \mathrm{Z} w x z \rightarrow \Phi w x y z) \\
& \& \operatorname{MAX}\left(\mathrm{W}, \mathrm{X} / \mathrm{C}_{3}, \mathrm{C}_{4}\right):\langle\mathrm{W}, \mathrm{X}\rangle \\
& \text { is a max. pair satisfying } \mathrm{C}_{3}, \mathrm{C}_{4} \\
& \& \operatorname{MAX}(\mathrm{Y}, \mathrm{Z} / \mathrm{IN}):\langle\mathrm{Y}, \mathrm{Z}\rangle \\
& \text { is a max. pair satisfying IN }] .
\end{aligned}
$$

Partial orderings are sometimes "unruly", and in some unruly cases a quantifier belongs to the same cluster as its governor. Thus, in (64), $\mathrm{Q}_{2} w$ and $\mathrm{Q}_{4} y$ belong to the same cluster. We interpret (64) in the usual way:

$$
\begin{aligned}
& \sum_{\mathrm{Q}_{2} w-\mathrm{Q}_{4} y}^{\mathrm{Q}_{1} v-\mathrm{Q}_{5} z} \overbrace{\Phi v w y z} \\
& ={ }_{\mathrm{Df}} \exists \mathrm{V}^{1} \mathrm{~W}^{1} \mathrm{X}^{3} \mathrm{Y}^{2} \mathrm{Z}^{4}\left[\mathrm{C}_{1}: \quad \mathrm{Q}_{1} v \mathrm{~V} v \& \mathrm{C}_{2}: \mathrm{Q}_{2} w \mathrm{~W} w\right. \\
& \& \mathrm{C}_{3}: \forall v w\left(\mathrm{~V} v \& \mathrm{~W} w \rightarrow \mathrm{Q}_{3} x \mathrm{X} v w x\right) \\
& \& \mathrm{C}_{4}: \forall w\left(\mathrm{~W} w \rightarrow \mathrm{Q}_{4} y \mathrm{Y} w y\right) \\
& \& \mathrm{C}_{5}: \forall v w y\left(\mathrm{~V} v \& \mathrm{Y} w y \rightarrow \mathrm{Q}_{5} z \mathrm{Z} v w y z\right) \\
& \text { \& IN: } \forall v w x y z(\mathrm{X} v w x \& \mathrm{Z} v w y z \rightarrow \Phi v w x y z) \\
& \& \operatorname{MAX}\left(\mathrm{V}, \mathrm{W}, \mathrm{Y} / \mathrm{C}_{3}, \mathrm{C}_{4}, \mathrm{C}_{5}\right):\langle\mathrm{V}, \mathrm{W}, \mathrm{Y}\rangle \\
& \text { is a max. triple satisfying } \mathrm{C}_{3}, \mathrm{C}_{4}, \mathrm{C}_{5} \\
& \& \operatorname{MAX}(\mathrm{X}, \mathrm{Z} / \mathrm{IN}):\langle\mathrm{Z}, \mathrm{X}\rangle \\
& \text { is a max. pair satisfying IN]. }
\end{aligned}
$$

To show that when $\mathrm{Q}_{1}-\mathrm{Q}_{5}$ are $\mathrm{M} \uparrow$ the maximality conditions can be omitted, we use the same method for extending a quintuple $\langle\mathrm{V}, \mathrm{W}, \mathrm{X}, \mathrm{Y}, \mathrm{Z}\rangle$ satisfying $C_{1}-I N$ to a quintuple $\left\langle V^{*}, W^{*}, X^{*}, Y^{*}, Z^{*}\right\rangle$ satisfying $C_{1}-$ $\operatorname{MAX}(\mathrm{X}, \mathrm{Z} / \mathrm{IN})$ as before, following the rule that relations associated with smaller quantifiers are extended before relations associated with larger quantifiers. In particular, we extend Y before we extend W. For example, we can extend the relations in this order: X, Z, Y,W, V. Now, 
suppose we have already extended $\mathrm{X}$ and $\mathrm{Z}$ so that $\left\langle\mathrm{V}, \mathrm{W}, \mathrm{X}^{*}, \mathrm{Y}, \mathrm{Z}^{*}\right\rangle$ satisfies $\mathrm{C}_{1}-\mathrm{IN}$ and $\mathrm{MAX}(\mathrm{X}, \mathrm{Z} / \mathrm{IN})$, and we now set out to extend $\mathrm{Y}$. $\mathrm{Y}$ appears in the antecedent of the quantified formula in $\mathrm{C}_{5}$ and in the consequent of the quantified formula in $\mathrm{C}_{4}$. But we do not have to worry about a clash: by assumption $\mathrm{Q}_{4}$ is $\mathrm{M} \uparrow$, hence any extension of $\mathrm{Y}$ satisfies $\mathrm{C}_{4}$, in particular the extension of $\mathrm{Y}$ to a maximal $\mathrm{Y}^{*}$ such that $\left\langle\mathrm{V}, \mathrm{Y}^{*}, \mathrm{Z}^{*}\right\rangle$ satisfies $\mathrm{C}_{5}$.

\section{Applications}

It is natural to look for applications in linguistics, mathematics, and computer science. In linguistics, (PGQ) removes the barrier on the complexity of branching generalized quantifications that the earlier definitions of the "basic cases" imposed. I will bring a few examples using, for the most part, the quantifier "two", understood as "exactly two". This choice allows me to construct graphic representations of the quantifications in question, and to express by means of a unary quantifier what otherwise would require binary quantifiers. (We can use the unary quantifier $! 2^{1}-$ "(Exactly 2) $)^{1 "}$ - to symbolize "Two A's are B's", but we need a binary quantifier, "Most" ${ }^{2}$ ", for "Most A's are B's").

Two boys have two toys and two friends who don't like their toys.

One reading of (7) is: "There are two boys who each has two toys and two friends, such that both his friends dislike both his toys". This reading is captured by:

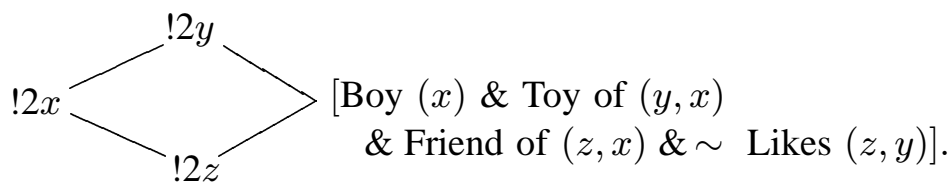

According to (PGQ), the definiens of (65) is (using obvious abbreviations):

$$
\begin{aligned}
& \exists \mathrm{X}^{1} \mathrm{Y}^{2} \mathrm{Z}^{2}[! 2 x \mathrm{X} x \& \mathrm{X} \text { is a max. set s.t. } \forall x(\mathrm{X} x \rightarrow ! 2 y \mathrm{Y} x y \\
& \quad \& ! 2 z \mathrm{Z} x z) \&\langle\mathrm{Y}, \mathrm{Z}\rangle \text { is a max. pair s.t. } \forall x y z(\mathrm{Y} x y \\
& \quad \& \mathrm{Z} x z \rightarrow \mathrm{B} x \& \mathrm{~T} y x \& \mathrm{~F} z x \& \sim \mathrm{L} z y)] .
\end{aligned}
$$

A typical situation in which (66) comes out true is one in which the extension of " $\mathrm{B} x \& \mathrm{~T} y x \& \mathrm{~F} z x \& \sim \mathrm{L} z y$ " includes exactly two maximal substructures of the form: 


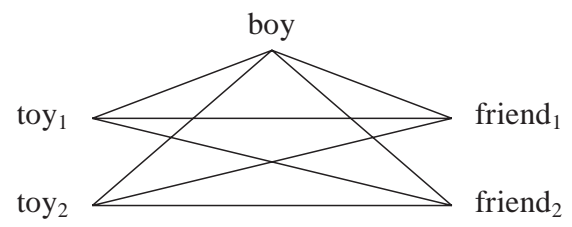

where the lines connecting friends to toys express the relation of dislike.

Two veterans have two friends and two foes who fought in the same two wars.

If we understand (8) as "There are two veterans who each has two friends and two foes each pair of whom fought in the same two wars", we will symbolize it as:

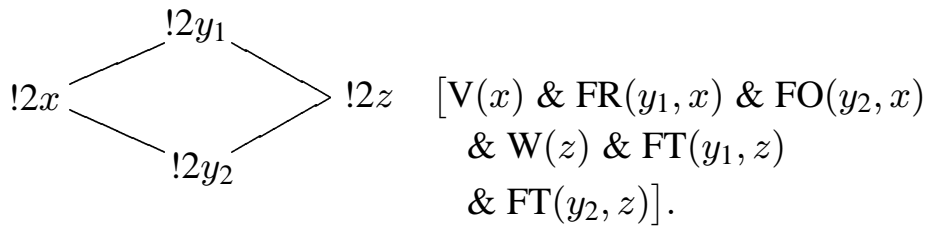

A typical situation in which (67) is true is one in which the extension of the quantified relation includes exactly two maximal sub-relations of the form:

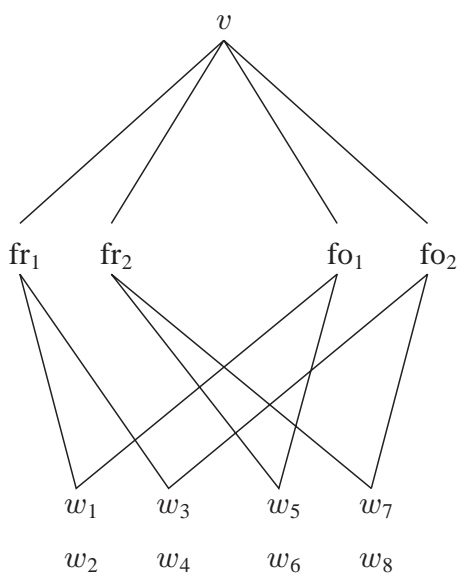

(Not all wars have to distinct, but there have to be at least two distinct wars.) We may also read (8) as "There are two veterans and two wars 
such that each veterans has two friends and two foes who fought in these two wars". This reading is symbolized by:

(68)

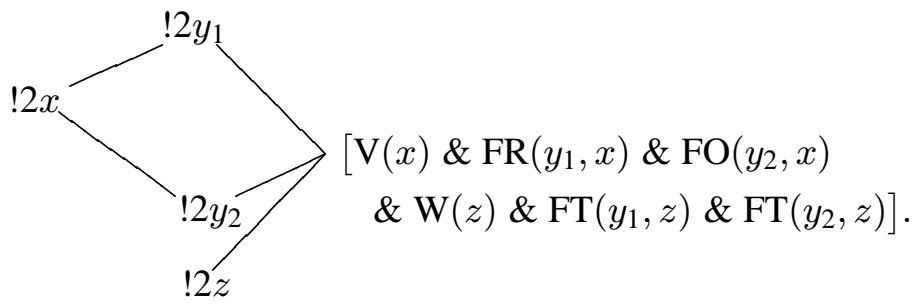

(69)

Two directors made several movies which were all "nixed" by the same two critics.

Under one reading (69) is symbolized by:

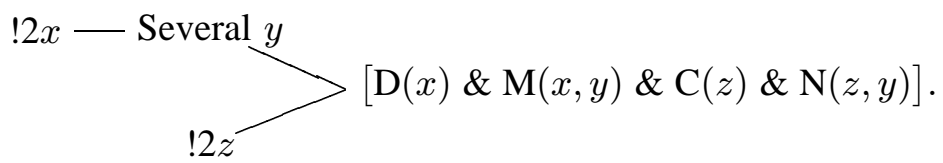

A typical situation where (70) is true is one in which the extension of the quantified relation includes a maximal subrelation of the form

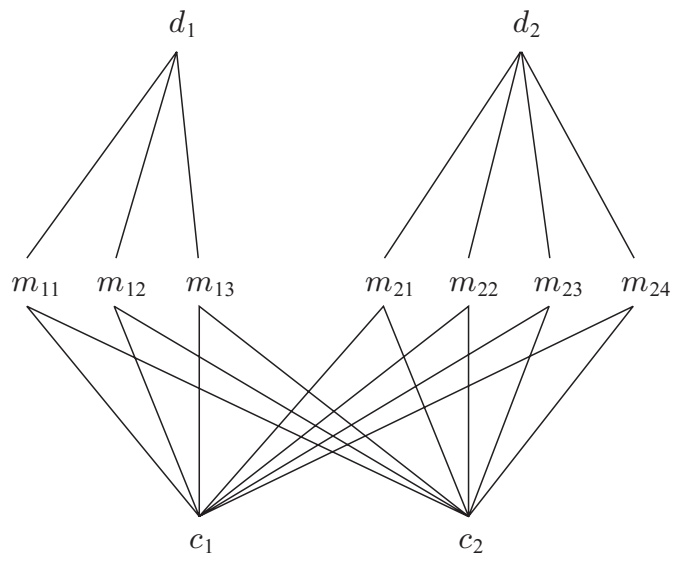

In mathematics, we can use (PGQ) to express such statements as $\alpha$ subsetes of $\mathrm{A}$ and $\beta$ subsets of $\mathrm{B}$ have no common elements (are pairwise disjoint), 
symbolized by

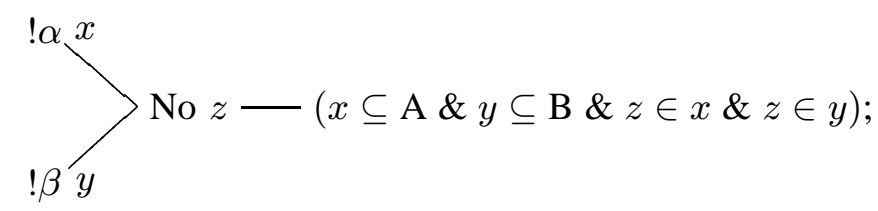

$$
\begin{aligned}
& \exists z-(x \in \mathrm{A} \& y \in \mathrm{B} \& f: \mathrm{A} \rightarrow \mathbb{R} \\
& \& g: \mathrm{B} \rightarrow \mathbb{R} \& f(x)-g(y)=z \& z \in \mathbb{Z})
\end{aligned}
$$

In computer science, it seems that some parallel processors can be encoded, semantically, by tree-like PGQs. Consider the simple case of a parallel processor which includes three units, $\mathrm{A}, \mathrm{B}$ and $\mathrm{C}$ as follows: unit $A$ is given two sets, $S_{1}$ and $S_{2}$, of $\alpha$ and $\beta$ numbers, respectively; unit $B$ is given two sets, $S_{3}$ and $S_{4}$, of $\gamma$ and $\delta$ numbers, respectively; unit A performs operation $f_{1}$ on each pair of numbers from $\mathrm{S}_{1}$ and $\mathrm{S}_{2}$; unit B performs operations $f_{2}$ on each pair of numbers from $S_{3}$ and $S_{4}$; and unit $\mathrm{C}$ performs operation $f_{3}$ on each pair of results from $\mathrm{A}$ and $\mathrm{B}$. We can describe such a processor by:

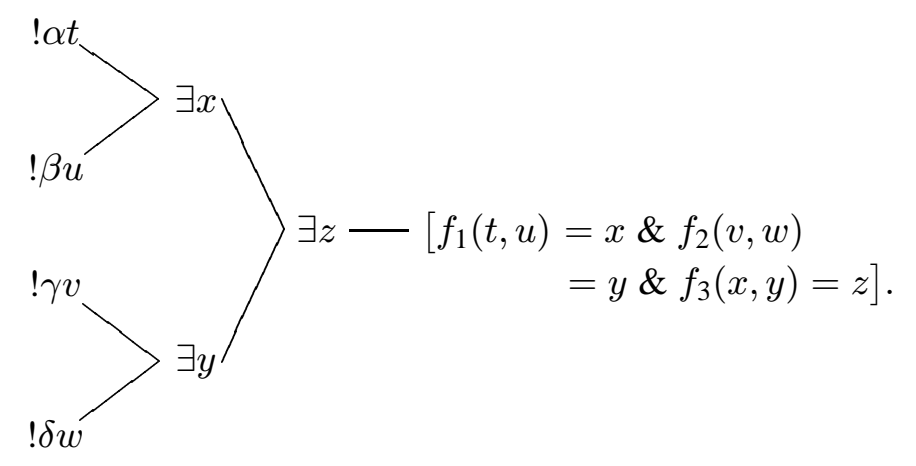

In "Ways of Branching Quantifiers" I pointed out that PGQ does not exhaust all the possibilities of non-linear languages, and I delineated a family of partially-ordered quantifier structures that includes the basic case of the branching form defined here as a particular instance. Many branching statements in natural and artificial languages require this wider conception of branching quantification, ${ }^{15}$ but the task of developing a general definition schema for the entire family of branching structures has yet to be accomplished. 


\section{NOTES}

${ }^{1}$ Recently, Martijn Spaan proposed a new definition of the basic case that combines the principles of Westerståhl's definition and mine. A critical discussion of Westerståhl and Sher appears in Schein (1993).

${ }^{2}$ I assume that the variables displayed in a given wff occur free in it and are the only variables occurring free in it. This assumption is, of course, not essential.

${ }^{3}$ In contrast with M. Mostowski (1987).

${ }^{4}$ I use " $>$ " where Walkoe uses " $<$ ".

${ }^{5}$ This is a simplification of sentence (51) in Hintikka (1973, p. 350).

${ }^{6}$ Many-place Mostowskian quantifiers were first presented in Lindström (1966). For two different approaches see Barwise and Cooper (1981) and Sher (1991a, Chapter 2).

${ }^{7}$ A partly different relational formulation of Henkin's definition (see stage ii) was given by Mostowski (1987). The difference reflects divergence in goals: Mostowski is interested in features of PSQ, I am interested in generalizing PSQ to PGQ.

${ }^{8}$ Here and in many of the example below, we can write the definiens in a more concise way, but for the sake of clarity I prefer to give the longer definition.

${ }^{9}$ Note that (44) also coincides with Barwise's (36) when $\mathrm{Q}_{1}$ is $\mathrm{M} \downarrow$ and with van Benthem's (42) in all cases. My definition of the basic case of a genuine PGQ, however, does not coincide with either Barwise's or van Benthem's definitions.

${ }^{10}$ Here I arrive at the maximality condition for the branching case through the familiar case of (22), therefore a special justification is not required. In Sher (1990a/1991a) I arrived at it directly for (27) and there I claimed the maximality condition was not ad hoc based on the observation that the branching prefix expresses a condition on a subset of the quantified relation, and generally, when we talk about sets it is maximal sets that we are interested in. Consider, for instance, "Three students passed the test". We intend this statement to be false when 10 students passed the test, but it will come out true if we do not require that ' $\exists ! 3$ ' be satisfied by a maximal set of students who passed the test. Consider also "No student passed the test" and "Two people live in America". Indeed, this explains why (22) involves a maximality condition.

${ }^{11}$ Suppose there is a pair, $\left\langle\mathrm{X}^{* *}, \mathrm{Y}^{* *}\right\rangle$ such that $\mathrm{X}^{*} \times \mathrm{Y}^{*} \varsubsetneqq \mathrm{X}^{* *} \times \mathrm{Y}^{* *} \subseteq \Phi$. Then either (i) $\mathrm{X}^{*} \times \mathrm{Y}^{*} \subsetneq \mathrm{X}^{* *} \times \mathrm{Y}^{*} \subseteq \Phi$ or (ii) $\mathrm{X}^{*} \times \mathrm{Y}^{*} \subsetneq \mathrm{X}^{*} \times \mathrm{Y}^{* *} \subseteq \Phi$. If (i), then $\mathrm{X}^{*} \neq \mathrm{X}^{* *}$; but also $\mathrm{X}^{* *} \times \mathrm{Y} \subseteq \Phi$, hence, by construction of $\mathrm{X}^{*}, \mathrm{X}^{*}=\mathrm{X}^{* *}$. Contradiction. If (ii), then $\mathrm{Y}^{*} \neq \mathrm{Y}^{* *}$; but also $\mathrm{X}^{*} \times \mathrm{Y}^{* *} \subseteq \Phi$, hence, by construction of $\mathrm{Y}^{*}, \mathrm{Y}^{*}=\mathrm{Y}^{* *}$. Contradiction.

${ }^{12}$ However, not all English $\mathrm{M} \downarrow$ branching quantifications are naturally interpreted by (46). Some are more naturally interpreted as independent quantifications, e.g., (38), and some exemplify other non-linear structures. See ending paragraph.

${ }^{13}$ Note that standard model theory does not distinguish between $X_{1} \times Y_{1}$ and $X_{2} \times Y_{2}$ where $\left|X_{1}\right| \neq\left|X_{2}\right|$ and $\left|Y_{1}\right|=\left|Y_{2}\right|=\phi$. As a result (6) is trivially true when the quantified relation, $\Phi$, is empty. When $\Phi$ is not empty, (6) is true iff a non-empty maximal Cartesian product with one domain satisfying "Most" and the other satisfying "Few" is included in $\Phi$. (The same applies to (40).)

${ }^{14}$ See (D) in Section I for an abbreviated formulation of the definiens.

${ }^{15}$ Examples (Sher 1990a, pp. 417-418 / 1991a, pp. 125-126):

(a) Most of my left hand gloves and most of my right hand gloves match one to one; 
(b) Most of my friends saw at least two of the same few Truffaut movies.

(c) By and large, no more than a few boys and a few girls all date one another.

\section{REFERENCES}

Barwise, J. 1979. “On Branching Quantifiers in English”, J. Philosophical Logic 8, 4780.

Barwise, J. and Cooper, R. 1981. "Generalized Quantifiers and Natural Language", Linguistics and Philosophy 4, 159-219.

Enderton, H.B. 1972. A Mathematical Introduction to Logic. New York, Academic Press. Henkin, L. 1959. "Some Remarks on Infinitely Long Formulas", Finitistic Methods, Proc. Symposium on Foundations of Math. Warsaw, 1961, 167-183.

Hintikka, J. 1973. "Quantifiers vs. Quantification Theory", Dialectica 27, 329-358.

Lindstöm, P. 1966. "First Order Predicate Logic with Generalized Quantifiers", Theoria 32, 186-195.

May, R. 1989. "Interpreting Logical Form”, Linguistics and Philosophy 12, 387-435.

Mostowski, A. 1957. "On a Generalization of Quantifiers", Fund. Mathematica 44, 12 36.

Mostowski, M. 1987. "The Relational Semantics for Branched Quantifiers”, Mathematical Logic and Its Applications. Ed. D.G. Skordev, New York, Plenum Press, 315-322.

Schein, B. 1993. Plurals and Events. Cambridge, MIT.

Sher, G. 1990a. "Ways of Branching Quantifiers", Linguistics and Philosophy 13, 393422. A slightly revised version appears in Sher 1991a, Ch. 5.

Sher, G. 1990b. "Two Approaches to Branching Quantification", Conference Proceedings: Theories of Partial Information, Center for Cog. Sc., U Texas, Austin.

Sher, G. 1991a. The Bounds of Logic: A Generalized Viewpoint. Cambridge, MIT.

Sher, G. 1991b. "Towards a General Definition of Partially-Ordered (Branching) Generalized Quantifiers", Abstract. 9th International Congress of Logic, Methodology and Philosophy of Science, Uppsala, Sweden, Vol. 3, p. 157.

Sher, G. 1994. "A General Definition of Partially-Ordered Generalized Quantification (PGQ)", Abstract. Journal of Symbolic Logic 59, 712-713.

van Benthem, J. 1989. "Polyadic Quantifiers", Linguistics and Philosophy 12, 437-464.

Westerståhl, D. 1987. "Branching Generalized Quantifiers and Natural Language", Generalized Quantifiers, Ed. P. Gärdenfors. Dordrecht, D. Reidel, 269-298.

Walkoe, W.J. Jr. 1970. "Finite Partially-Ordered Quantification", Journal of Symbolic Logic 35, 535-555.

Philosophy Department, 9500 Gilman Drive, 0119,

University of California at San Diego,

La Jolla, CA 92093-0119, U.S.A. 